\title{
Existence of Solutions for a Weighted $p(t)$-Laplacian Impulsive Integrodifferential System with Multipoint and Integral Boundary Value Conditions
}

\author{
Rong Dong, ${ }^{1}$ Yunrui Guo, ${ }^{2}$ Yuanzhang Zhao, ${ }^{3}$ and Qihu Zhang ${ }^{1}$ \\ ${ }^{1}$ Department of Mathematics and Information Science, Zhengzhou University of Light Industry, \\ Zhengzhou, Henan 450002, China \\ 2 Department of Mathematics, Henan Institute of Science and Technology, Xinxiang, Henan 453003, China \\ ${ }^{3}$ Department of Mathematics, Ocean University of China, Qingdao, Shandong 266071, China
}

Correspondence should be addressed to Qihu Zhang, zhangqh1999@yahoo.com.cn

Received 5 October 2010; Accepted 15 December 2010

Academic Editor: Jewgeni Dshalalow

Copyright (C) 2010 Rong Dong et al. This is an open access article distributed under the Creative Commons Attribution License, which permits unrestricted use, distribution, and reproduction in any medium, provided the original work is properly cited.

By the Leray-Schauder's degree, the existence of solutions for a weighted $p(t)$-Laplacian impulsive integro-differential system with multi-point and integral boundary value conditions is considered. The sufficient results for the existence are given under the resonance and nonresonance cases, respectively. Moreover, we get the existence of nonnegative solutions at nonresonance.

\section{Introduction}

In this paper, we consider the existence of solutions for the following weighted $p(t)$-Laplacian integrodifferential system:

$$
-\Delta_{p(t)} u+f\left(t, u,(w(t))^{1 /(p(t)-1)} u^{\prime}, S(u), T(u)\right)=0, \quad t \in(0,1), t \neq t_{i}
$$

where $u:[0,1] \rightarrow \mathbb{R}^{N}, f(\cdot, \cdot, \cdot, \cdot, \cdot):[0,1] \times \mathbb{R}^{N} \times \mathbb{R}^{N} \times \mathbb{R}^{N} \times \mathbb{R}^{N} \rightarrow \mathbb{R}^{N}, t_{i} \in(0,1), i=1, \ldots, k$, with the following impulsive boundary value conditions

$$
\lim _{t \rightarrow t_{i}^{+}} u(t)-\lim _{t \rightarrow t_{i}^{-}} u(t)=A_{i}\left(\lim _{t \rightarrow t_{i}^{-}} u(t), \lim _{t \rightarrow t_{i}^{-}}(w(t))^{1 /(p(t)-1)} u^{\prime}(t)\right), \quad i=1, \ldots, k
$$




$$
\begin{gathered}
\lim _{t \rightarrow t_{i}^{+}} w(t)\left|u^{\prime}\right|^{p(t)-2} u^{\prime}(t)-\lim _{t \rightarrow t_{i}^{-}} w(t)\left|u^{\prime}\right|^{p(t)-2} u^{\prime}(t) \\
=B_{i}\left(\lim _{t \rightarrow t_{i}^{-}} u(t), \lim _{t \rightarrow t_{i}^{-}}(w(t))^{1 /(p(t)-1)} u^{\prime}(t)\right), \quad i=1, \ldots, k, \\
\lim _{t \rightarrow 0^{+}} w(t)\left|u^{\prime}\right|^{p(t)-2} u^{\prime}(t)=\sum_{\ell=1}^{m-2} \alpha_{\ell} \lim _{t \rightarrow \eta_{e}^{-}} w(t)\left|u^{\prime}\right|^{p(t)-2} u^{\prime}(t), \quad u(0)=\int_{0}^{1} g(t) u(t) d t,
\end{gathered}
$$

where $p \in C([0,1], \mathbb{R})$ and $p(t)>1,-\Delta_{p(t)} u:=-\left(w(t)\left|u^{\prime}\right|^{p(t)-2} u^{\prime}\right)^{\prime}$ is called the weighted $p(t)$-Laplacian; $0<t_{1}<t_{2}<\cdots<t_{k}<1,0<\eta_{1}<\cdots<\eta_{m-2}<1 ; \alpha_{\ell} \geq 0$, $(\ell=1, \ldots$, $m-2)$ and $0 \leq \sum_{\ell=1}^{m-2} \alpha_{\ell} \leq 1 ; g \in L^{1}[0,1]$ is nonnegative, $\int_{0}^{1} g(t) d t=\sigma$ with $\sigma \in[0,1]$; $A_{i}, B_{i} \in C\left(\mathbb{R}^{N} \times \mathbb{R}^{N}, \mathbb{R}^{N}\right) ; T$ and $S$ are linear operators defined by $(T u)(t)=\int_{0}^{t} k_{*}(t, s) u(s) d s$, $(S u)(t)=\int_{0}^{1} h_{*}(t, s) u(s) d s, t \in[0,1]$, where $k_{*}, h_{*} \in C([0,1] \times[0,1], \mathbb{R})$.

If $\sum_{\ell=1}^{m-2} \alpha_{\ell}<1$ and $\sigma<1$, we say the problem is nonresonant, but if $\sum_{\ell=1}^{m-2} \alpha_{\ell}=1$ and $\sigma=1$, we say the problem is resonant.

Throughout the paper, $o(1)$ means function which uniformly convergent to 0 (as $n \rightarrow$ $+\infty)$; for any $v \in \mathbb{R}^{N}, v^{j}$ will denote the $j$ th component of $v$; the inner product in $\mathbb{R}^{N}$ will be denoted by $\langle\cdot, \cdot\rangle ;|\cdot|$ will denote the absolute value and the Euclidean norm on $\mathbb{R}^{N}$. Denote $J=[0,1], J^{\prime}=(0,1) \backslash\left\{t_{1}, \ldots, t_{k}\right\}, J_{0}=\left[t_{0}, t_{1}\right], J_{i}=\left(t_{i}, t_{i+1}\right], i=1, \ldots, k$, where $t_{0}=0, t_{k+1}=1$. Denote $J_{i}^{o}$ the interior of $J_{i}, i=0,1, \ldots, k$. Let

$$
\operatorname{PC}\left(J, \mathbb{R}^{N}\right)=\left\{\begin{array}{l|l}
x: J \longrightarrow \mathbb{R}^{N} & \begin{array}{l}
x \in C\left(J_{i}, \mathbb{R}^{N}\right), i=0,1, \ldots, k, \\
\lim _{t \rightarrow t_{i}^{+}} x(t) \text { exists for } i=1, \ldots, k
\end{array}
\end{array}\right\},
$$

$w \in \mathrm{PC}(J, \mathbb{R})$ satisfies $0<w(t)$, for all $t \in(0,1) \backslash\left\{t_{1}, \ldots, t_{k}\right\}$, and $(w(t))^{-1 /(p(t)-1)} \in L^{1}(0,1)$

$$
\operatorname{PC}^{1}\left(J, \mathbb{R}^{N}\right)=\left\{\begin{array}{l|l}
x \in \operatorname{PC}\left(J, \mathbb{R}^{N}\right) & \begin{array}{l}
x^{\prime} \in C\left(J_{i}^{o}, \mathbb{R}^{N}\right), \lim _{t \rightarrow t_{i}^{+}}(w(t))^{1 /(p(t)-1)} x^{\prime}(t), \\
\lim _{t \rightarrow t_{i+1}^{-}}(w(t))^{1 /(p(t)-1)} x^{\prime}(t) \text { exists for } i=0,1, \ldots, k
\end{array}
\end{array}\right\} .
$$

For any $x=\left(x^{1}, \ldots, x^{N}\right) \in \mathrm{PC}\left(J, \mathbb{R}^{N}\right)$, denote $\left|x^{i}\right|_{0}=\sup \left\{\left|x^{i}(t)\right| \mid t \in J^{\prime}\right\}$. Obviously, $\operatorname{PC}\left(J, \mathbb{R}^{N}\right)$ is a Banach space with the norm $\|x\|_{0}=\left(\sum_{i=1}^{N}\left|x^{i}\right|_{0}^{2}\right)^{1 / 2}$, and $\operatorname{PC}^{1}\left(J, \mathbb{R}^{N}\right)$ is a Banach space with the norm $\|x\|_{1}=\|x\|_{0}+\left\|(w(t))^{1 /(p(t)-1)} x^{\prime}\right\|_{0}$. Denote $L^{1}=L^{1}\left(J, \mathbb{R}^{N}\right)$ with the norm $\|x\|_{L^{1}}=\left(\sum_{i=1}^{N}\left|x^{i}\right|_{L^{1}}^{2}\right)^{1 / 2}$, for all $x \in L^{1}$, where $\left|x^{i}\right|_{L^{1}}=\int_{0}^{1}\left|x^{i}(t)\right| d t$. denote

For simplicity, we denote $\mathrm{PC}\left(J, \mathbb{R}^{N}\right)$ and $\operatorname{PC}^{1}\left(J, \mathbb{R}^{N}\right)$ by $\mathrm{PC}$ and $\mathrm{PC}^{1}$, respectively, and

$$
\begin{gathered}
u\left(t_{i}^{+}\right)=\lim _{t \rightarrow t_{i}^{+}} u(t), \quad u\left(t_{i}^{-}\right)=\lim _{t \rightarrow t_{i}^{-}} u(t), \\
w(0)\left|u^{\prime}\right|^{p(0)-2} u^{\prime}(0)=\lim _{t \rightarrow 0^{+}} w(t)\left|u^{\prime}\right|^{p(t)-2} u^{\prime}(t), \\
w(1)\left|u^{\prime}\right|^{p(1)-2} u^{\prime}(1)=\lim _{t \rightarrow 1^{-}} w(t)\left|u^{\prime}\right|^{p(t)-2} u^{\prime}(t),
\end{gathered}
$$




$$
\begin{gathered}
A_{i}=A_{i}\left(\lim _{t \rightarrow t_{i}^{-}} u(t), \lim _{t \rightarrow t_{i}^{-}}(w(t))^{1 /(p(t)-1)} u^{\prime}(t)\right), \quad i=1, \ldots, k, \\
B_{i}=B_{i}\left(\lim _{t \rightarrow t_{i}^{-}} u(t), \lim _{t \rightarrow t_{i}^{-}}(w(t))^{1 /(p(t)-1)} u^{\prime}(t)\right), \quad i=1, \ldots, k .
\end{gathered}
$$

In recent years, there has been an increasing interest in the study of differential equations with nonstandard $p(t)$-growth conditions. These problems have many interesting applications (see [1-4]). Many results have been obtained on these kinds of problems, for example [5-17]. If $p(t) \equiv p$ (a constant), (1.1)-(1.4) becomes the well known $p$-Laplacian problem. If $p(t)$ is a general function, one can see easily that $-\Delta_{p(t)} c u \neq c^{p(t)}\left(-\Delta_{p(t)} u\right)$ in general, while $-\Delta_{p} c u=c^{p}\left(-\Delta_{p} u\right)$, so $-\Delta_{p(t)}$ represents a non-homogeneity and possesses more nonlinearity, thus $-\Delta_{p(t)}$ is more complicated than $-\Delta_{p}$. For example, we have the following.

(a) In general, the infimum $\lambda_{p(x)}$ of eigenvalues for the $p(x)$-Laplacian Dirichlet problems is zero, and $\lambda_{p(x)}>0$ only under some special conditions (see [10]). When $\Omega \subset \mathbb{R}(N=1)$ is an interval, the results in [10] show that $\lambda_{p(x)}>0$ if and only if $p(x)$ is monotone. But the property of $\lambda_{p}>0$ is very important in the study of $p$-Laplacian problems, for example, in [18], the authors use this property to deal with the existence of solutions.

(b) If $w(t) \equiv 1$ and $p(t) \equiv p$ (a constant) and $-\Delta_{p} u>0$, then $u$ is concave, this property is used extensively in the study of one-dimensional $p$-Laplacian problems (see [19]), but it is invalid for $-\Delta_{p(t)}$. It is another difference between $-\Delta_{p}$ and $-\Delta_{p(t)}$.

Recently, there are many works devoted to the existence of solutions to the Laplacian impulsive differential equation boundary value problems, for example [20-28]. Many methods had been applied to deal with these problems, for example sub-super-solution method, fixed point theorem, monotone iterative method, coincidence degree, variational principles (see [29]), and so forth. Because of the nonlinearity of $-\Delta_{p}$, results about the existence of solutions for $p$-Laplacian impulsive differential equation boundary value problems are rare (see [30]). In [31], using coincidence degree method, the present author investigate the existence of solutions for $p(r)$-Laplacian impulsive differential equation with multipoint boundary value conditions. Integral boundary conditions for evolution problems have various applications in chemical engineering, thermoelasticity, underground water flow and population dynamics, there are many papers on the differential equations with integral boundary value problems, for example, [32-35].

In this paper, when $p(t)$ is a general function, we investigate the existence of solutions and nonnegative solutions for the weighted $p(t)$-Laplacian impulsive integrodifferential system with multipoint and integral boundary value conditions. Our results contain both the cases of resonance and nonresonance, and the method is based upon Leray-Schauder's degree. Moreover, this paper will consider the existence of (1.1) with (1.2), (1.4) and the following impulsive condition:

$$
\begin{aligned}
& \lim _{t \rightarrow t_{i}^{+}}(w(t))^{1 /(p(t)-1)} u^{\prime}(t)-\lim _{t \rightarrow t_{i}^{-}}(w(t))^{1 /(p(t)-1)} u^{\prime}(t) \\
& \quad=D_{i}\left(\lim _{t \rightarrow t_{i}^{-}} u(t), \lim _{t \rightarrow t_{i}^{-}}(w(t))^{1 /(p(t)-1)} u^{\prime}(t)\right), \quad i=1, \ldots, k,
\end{aligned}
$$


where $D_{i} \in C\left(\mathbb{R}^{N} \times \mathbb{R}^{N}, \mathbb{R}^{N}\right)$, the impulsive condition (1.8) is called linear impulsive condition (LI for short), and (1.3) is called nonlinear impulsive condition (NLI for short). In generaly, $p$-Laplacian impulsive problems have two kinds of impulsive conditions, that is, LI and NLI.

Let $N \geq 1$, the function $f: J \times \mathbb{R}^{N} \times \mathbb{R}^{N} \times \mathbb{R}^{N} \times \mathbb{R}^{N} \rightarrow \mathbb{R}^{N}$ is assumed to be Caratheodory, by this we mean the following:

(i) for almost every $t \in J$ the function $f(t, \cdot, \cdot, \cdot, \cdot)$ is continuous;

(ii) for each $(x, y, s, z) \in \mathbb{R}^{N} \times \mathbb{R}^{N} \times \mathbb{R}^{N} \times \mathbb{R}^{N}$ the function $f(\cdot, x, y, s, z)$ is measurable on $J$;

(iii) for each $R>0$ there is a $\alpha_{R} \in L^{1}(J, \mathbb{R})$ such that, for almost every $t \in J$ and every $(x, y, s, z) \in \mathbb{R}^{N} \times \mathbb{R}^{N} \times \mathbb{R}^{N} \times \mathbb{R}^{N}$ with $|x| \leq R,|y| \leq R,|s| \leq R,|z| \leq R$, one has

$$
|f(t, x, y, s, z)| \leq \alpha_{R}(t)
$$

We say a function $u: J \rightarrow \mathbb{R}^{N}$ is a solution of (1.1) if $u \in \mathrm{PC}^{1}$ with $w(t)\left|u^{\prime}\right|^{p(t)-2} u^{\prime}$ absolutely continuous on $J_{i}^{o}, i=0,1, \ldots, k$, which satisfies (1.1) a.e. on $J$.

In this paper, we always use $C_{i}$ to denote positive constants, if it cannot lead to confusion. Denote

$$
z^{-}=\inf _{t \in J} z(t), \quad z^{+}=\sup _{t \in J} z(t), \quad \text { for any } z \in \operatorname{PC}(J, \mathbb{R}) .
$$

We say $f$ satisfies sub- $\left(p^{-}-1\right)$ growth condition, if $f$ satisfies

$$
\lim _{|u|+|v|+|s|+|z| \rightarrow+\infty} \frac{f(t, u, v, s, z)}{(|u|+|v|+|s|+|z|)^{q(t)-1}}=0, \quad \text { for } t \in J \text { uniformly, }
$$

where $q(t) \in \operatorname{PC}(J, \mathbb{R})$, and $1<q^{-} \leq q^{+}<p^{-}$.

This paper is organized as four sections. In Section 2, we present some preliminary and give the operator equation which has the same solutions of (1.1)-(1.4). In Section 3, we give the existence of solutions and nonnegative solutions for system (1.1)-(1.4) at nonresonance. Finally, in Section 4, we give the existence of solutions for system (1.1)-(1.4) at resonance.

\section{Preliminary}

For any $(t, x) \in J \times \mathbb{R}^{N}$, denote $\varphi(t, x)=|x|^{p(t)-2} x$. Obviously, $\varphi$ has the following properties.

Lemma 2.1 (see [31]). $\varphi$ is a continuous function and satisfies the following.

(i) For any $t \in[0,1], \varphi(t, \cdot)$ is strictly monotone, satisfying

$$
\left\langle\varphi\left(t, x_{1}\right)-\varphi\left(t, x_{2}\right), x_{1}-x_{2}\right\rangle>0, \text { for any } x_{1}, x_{2} \in \mathbb{R}^{N}, x_{1} \neq x_{2} \text {. }
$$

(ii) There exists a function $\alpha:[0,+\infty) \rightarrow[0,+\infty), \alpha(s) \rightarrow+\infty$ as $s \rightarrow+\infty$, such that

$$
\langle\varphi(t, x), x\rangle \geq \alpha(|x|)|x|, \quad \forall x \in \mathbb{R}^{N} .
$$


It is well known that $\varphi(t, \cdot)$ is an homeomorphism from $\mathbb{R}^{N}$ to $\mathbb{R}^{N}$ for any fixed $t \in J$. Denote

$$
\varphi^{-1}(t, x)=|x|^{(2-p(t)) /(p(t)-1)} x, \quad \text { for } x \in \mathbb{R}^{N} \backslash\{0\}, \varphi^{-1}(t, 0)=0, \forall t \in J .
$$

It is clear that $\varphi^{-1}(t, \cdot)$ is continuous and sends bounded sets to bounded sets.

In this section, we will do some preparation and give the operator equation which has the same solutions of (1.1)-(1.4). At first, let us now consider the following simple impulsive problem with boundary value condition (1.4)

$$
\begin{gathered}
-\Delta_{p(t)} u+f(t)=0, \quad t \in(0,1), \quad t \neq t_{i}, \\
\lim _{t \rightarrow t_{i}^{+}} u(t)-\lim _{t \rightarrow t_{i}^{-}} u(t)=a_{i}, \quad i=1, \ldots, k, \\
\lim _{t \rightarrow t_{i}^{+}} w(t)\left|u^{\prime}\right|^{p(t)-2} u^{\prime}(t)-\lim _{t \rightarrow t_{i}^{-}} w(t)\left|u^{\prime}\right|^{p(t)-2} u^{\prime}(t)=b_{i}, \quad i=1, \ldots, k,
\end{gathered}
$$

where $a_{i}, b_{i} \in \mathbb{R}^{N} ; f \in L^{1}$.

We will discuss (2.4) with (1.4) in the cases of resonance and nonresonance, respectively.

\subsection{The Case of Nonresonance}

Suppose $0 \leq \sum_{\ell=1}^{m-2} \alpha_{\ell}<1$ and $0 \leq \sigma<1$. If $u$ is a solution of (2.4) with (1.4), we have

$$
w(t) \varphi\left(t, u^{\prime}(t)\right)=w(0) \varphi\left(0, u^{\prime}(0)\right)+\sum_{t_{i}<t} b_{i}+\int_{0}^{t} f(s) d s, \quad \forall t \in J^{\prime} .
$$

Denote $a=\left(a_{1}, \ldots, a_{k}\right) \in \mathbb{R}^{k N}, b=\left(b_{1}, \ldots, b_{k}\right) \in \mathbb{R}^{k N}, \rho_{1}=w(0) \varphi\left(0, u^{\prime}(0)\right)$. It is easy to see that $\rho_{1}$ is dependent on $a, b$ and $f(t)$. Define operator $F: L^{1} \rightarrow \mathrm{PC}$ as

$$
F(f)(t)=\int_{0}^{t} f(s) d s, \quad \forall t \in J, \forall f \in L^{1}
$$

By solving for $u^{\prime}$ in (2.5) and integrating, we find

$$
u(t)=u(0)+\sum_{t_{i}<t} a_{i}+F\left\{\varphi^{-1}\left[t,(w(t))^{-1}\left(\rho_{1}+\sum_{t_{i}<t} b_{i}+F(f)(t)\right)\right]\right\}(t), \quad \forall t \in J,
$$

which together with the boundary value condition (1.4) implies

$$
\begin{gathered}
\rho_{1}=\frac{\left\{\sum_{\ell=1}^{m-2} \alpha_{\ell}\left[\sum_{t_{i}<\eta_{\ell}} b_{i}+F(f)\left(\eta_{\ell}\right)\right]\right\}}{1-\sum_{\ell=1}^{m-2} \alpha_{\ell}}, \\
u(0)=\frac{1}{(1-\sigma)} \int_{0}^{1} g(t)\left\{F\left\{\varphi^{-1}\left[t,(w(t))^{-1}\left(\rho_{1}+\sum_{t_{i}<t} b_{i}+F(f)(t)\right)\right]\right\}(t)+\sum_{t_{i}<t} a_{i}\right\} d t .
\end{gathered}
$$


Denote $W=\mathbb{R}^{2 k N} \times L^{1}$ with the norm $\|\omega\|=\sum_{i=1}^{k}\left|a_{i}\right|+\sum_{i=1}^{k}\left|b_{i}\right|+\|h\|_{L^{1}}$, for all $\omega=$ $(a, b, h) \in W$, then $W$ is a Banach space.

We define $\widetilde{\rho_{1}}: W \rightarrow \mathbb{R}^{N}$ as

$$
\widetilde{\rho_{1}}(\omega)=\frac{\left\{\sum_{\ell=1}^{m-2} \alpha_{\ell}\left[\sum_{t_{i}<\eta_{\ell}} b_{i}+F(h)(\eta \ell)\right]\right\}}{1-\sum_{\ell=1}^{m-2} \alpha_{\ell}}, \quad \forall \omega=(a, b, h) \in W,
$$

then $\widetilde{\rho_{1}}(\cdot)$ is continuous. Throughout the paper, we denote $E=\int_{0}^{1}(w(t))^{-1 /(p(t)-1)} d t$. It is easy to see the following.

Lemma 2.2. The function $\widetilde{\rho_{1}}: W \rightarrow \mathbb{R}^{N}$ is continuous and sends bounded sets to bounded sets. Moreover, for any $\omega=(a, b, h) \in W$, we have

$$
\left|\widetilde{\rho_{1}}(\omega)\right| \leq \frac{\sum_{\ell=1}^{m-2} \alpha_{\ell}\left[\sum_{i=1}^{k}\left|b_{i}\right|+\|h\|_{L^{1}}\right]}{1-\sum_{\ell=1}^{m-2} \alpha_{\ell}}
$$

We denote $N_{f}(u):[0,1] \times P C^{1} \rightarrow L^{1}$ the Nemytskii operator associated to $f$ defined by

$$
N_{f}(u)(t)=f\left(t, u(t),(w(t))^{1 /(p(t)-1)} u^{\prime}(t), S(u), T(u)\right) \text {, a.e. on } J \text {. }
$$

We define $\rho_{1}: P C^{1} \rightarrow \mathbb{R}^{N}$ as

$$
\rho_{1}(u)=\widetilde{\rho_{1}}\left(A, B, N_{f}\right)(u),
$$

where $A=\left(A_{1}, \ldots, A_{k}\right), B=\left(B_{1}, \ldots, B_{k}\right)$.

It is clear that $\rho_{1}(\cdot)$ is continuous and sends bounded sets of $\mathrm{PC}^{1}$ to bounded sets of $\mathbb{R}^{N}$, and hence it is compact continuous.

If $u$ is a solution of (2.4) with (1.4), we have

$$
u(t)=u(0)+\sum_{t_{i}<t} a_{i}+F\left\{\varphi^{-1}\left[t,(w(t))^{-1}\left(\widetilde{\rho_{1}}(\omega)+\sum_{t_{i}<t} b_{i}+F(f)(t)\right)\right]\right\}(t), \quad \forall t \in[0,1]
$$

For fixed $a, b \in \mathbb{R}^{k N}$, we define $K_{(a, b)}: L^{1} \rightarrow \mathrm{PC}^{1}$ as

$$
K_{(a, b)}(h)(t)=F\left\{\varphi^{-1}\left[t,(w(t))^{-1}\left(\widetilde{\rho_{1}}(a, b, h)+\sum_{t_{i}<t} b_{i}+F(h)(t)\right)\right]\right\}(t), \quad \forall t \in J .
$$




$$
\begin{aligned}
& \text { Define } K_{1}: \mathrm{PC}^{1} \rightarrow \mathrm{PC}^{1} \text { as } \\
& K_{1}(u)(t)=F\left\{\varphi^{-1}\left[t,(w(t))^{-1}\left(\rho_{1}(u)+\sum_{t_{i}<t} B_{i}+F\left(N_{f}(u)\right)(t)\right)\right]\right\}(t), \quad \forall t \in J .
\end{aligned}
$$

Lemma 2.3. (i) The operator $K_{(a, b)}$ is continuous and sends equiintegrable sets in $L^{1}$ to relatively compact sets in $P C^{1}$. in $P C^{1}$.

(ii) The operator $K_{1}$ is continuous and sends bounded sets in $P C^{1}$ to relatively compact sets

Proof. (i) It is easy to check that $K_{(a, b)}(h)(\cdot) \in \mathrm{PC}^{1}$, for all $h \in L^{1}$, for all $a, b \in \mathbb{R}^{k N}$. Since $(w(t))^{-1 /(p(t)-1)} \in L^{1}$ and

$$
K_{(a, b)}(h)^{\prime}(t)=\varphi^{-1}\left[t,(w(t))^{-1}\left(\widetilde{\rho_{1}}(a, b, h)+\sum_{t_{i}<t} b_{i}+F(h)\right)\right], \quad \forall t \in[0,1]
$$

it is easy to check that $K_{(a, b)}(\cdot)$ is a continuous operator from $L^{1}$ to $\mathrm{PC}^{1}$.

Let $U$ be an equiintegrable set in $L^{1}$, then there exists $\tau \in L^{1}$, such that

$$
|u(t)| \leq \tau(t) \quad \text { a.e. in } J \text {, for any } u \in L^{1} .
$$

We want to show that $\overline{K_{(a, b)}(U)} \subset \mathrm{PC}^{1}$ is a compact set.

Let $\left\{u_{n}\right\}$ be a sequence in $K_{(a, b)}(U)$, then there exists a sequence $\left\{h_{n}\right\} \in U$ such that $u_{n}=K_{(a, b)}\left(h_{n}\right)$. For any $t_{1}, t_{2} \in J$, we have

$$
\left|F\left(h_{n}\right)\left(t_{1}\right)-F\left(h_{n}\right)\left(t_{2}\right)\right|=\left|\int_{0}^{t_{1}} h_{n}(t) d t-\int_{0}^{t_{2}} h_{n}(t) d t\right|=\left|\int_{t_{1}}^{t_{2}} h_{n}(t) d t\right| \leq\left|\int_{t_{1}}^{t_{2}} \tau(t) d t\right| .
$$

Hence the sequence $\left\{F\left(h_{n}\right)\right\}$ is uniformly bounded and equicontinuous. By AscoliArzela theorem, there exists a subsequence of $\left\{F\left(h_{n}\right)\right\}$ (which we rename the same) which is convergent in PC. According to the bounded continuous of the operator $\widetilde{\rho_{1}}$, we can choose a subsequence of $\left\{\widetilde{\rho_{1}}\left(a, b, h_{n}\right)+F\left(h_{n}\right)\right\}$ (which we still denote by $\left.\left\{\widetilde{\rho_{1}}\left(a, b, h_{n}\right)+F\left(h_{n}\right)\right\}\right)$ which is convergent in PC, then $w(t)^{1 /(p(t)-1)} K_{(a, b)}\left(h_{n}\right)^{\prime}(t)=\varphi^{-1}\left(t, \widetilde{\rho_{1}}\left(a, b, h_{n}\right)+\sum_{t_{i}<t} b_{i}+F\left(h_{n}\right)\right)$ is convergent in PC.

Since

$$
K_{(a, b)}\left(h_{n}\right)(t)=F\left\{\varphi^{-1}\left[t,(w(t))^{-1}\left(\widetilde{\rho_{1}}\left(a, b, h_{n}\right)+\sum_{t_{i}<t} b_{i}+F\left(h_{n}\right)\right)\right]\right\}(t), \quad \forall t \in[0,1]
$$

it follows from the continuity of $\varphi^{-1}$ and the integrability of $w(t)^{-1 /(p(t)-1)}$ in $L^{1}$ that $K_{(a, b)}\left(h_{n}\right)$ is convergent in PC. Thus $\left\{u_{n}\right\}$ is convergent in $\mathrm{PC}^{1}$.

(ii) It is easy to see from (i) and Lemma 2.2.

This completes the proof. 
Let us define $P_{1}: \mathrm{PC}^{1} \rightarrow \mathrm{PC}^{1}$ as $P_{1}(u)=\left\{\int_{0}^{1} g(t)\left[K_{1}(u)(t)+\sum_{t_{i}<t} A_{i}\right] d t\right\} /(1-\sigma)$.

It is easy to see that $P_{1}$ is compact continuous.

Lemma 2.4. Suppose $0 \leq \sum_{\ell=1}^{m-2} \alpha_{\ell}<1$ and $0 \leq \sigma<1$, then $u$ is a solution of (1.1)-(1.4) if and only if $u$ is a solution of the following abstract operator equation

$$
u=P_{1}(u)+\sum_{t_{i}<t} A_{i}+K_{1}(u)
$$

Proof. Suppose $u$ is a solution of (1.1)-(1.4). From the definition of $\rho_{1}(\cdot)$ and $P_{1}(\cdot)$, similar to the discussion before Lemma 2.2, we know that $u$ is a solution of (2.20).

Conversely, if $u$ is a solution of (2.20), then (1.2) is satisfied.

From (2.20), we have

$$
\begin{gathered}
w(t) \varphi\left(t, u^{\prime}(t)\right)=\rho_{1}(u)+\sum_{t_{i}<t} B_{i}+F\left(N_{f}(u)\right)(t), \quad t \in(0,1), t \neq t_{i}, \\
\left(w(t) \varphi\left(t, u^{\prime}\right)\right)^{\prime}=N_{f}(u)(t), \quad t \in(0,1), t \neq t_{i} .
\end{gathered}
$$

It follows from (2.21) that (1.3) is satisfied.

From (2.21) and the definition of $\rho_{1}$, we have

$$
\lim _{t \rightarrow 0^{+}} w(t)\left|u^{\prime}\right|^{p(t)-2} u^{\prime}(t)=\sum_{\ell=1}^{m-2} \alpha_{\ell} \lim _{t \rightarrow \eta_{\ell}^{-}} w(t)\left|u^{\prime}\right|^{p(t)-2} u^{\prime}(t)
$$

From (2.20) and the definition of $P_{1}$, it is easy to check that

$$
u(0)=\int_{0}^{1} g(t) u(t) d t
$$

It follows from (2.22) and (2.23) that (1.4) is satisfied.

Hence $u$ is a solutions of (1.1)-(1.4). This completes the proof.

\subsection{The Case of Resonance}

Suppose $\sum_{\ell=1}^{m-2} \alpha_{\ell}=1$ and $\sigma=1$. If $u$ is a solution of (2.4) with (1.4), we have

$$
w(t) \varphi\left(t, u^{\prime}(t)\right)=w(0) \varphi\left(0, u^{\prime}(0)\right)+\sum_{t_{i}<t} b_{i}+\int_{0}^{t} f(s) d s, \quad \forall t \in J^{\prime} .
$$

Denote $a=\left(a_{1}, \ldots, a_{k}\right) \in \mathbb{R}^{k N}, b=\left(b_{1}, \ldots, b_{k}\right) \in \mathbb{R}^{k N}, \rho_{2}=w(0) \varphi\left(0, u^{\prime}(0)\right)$. It is easy to see that $\rho_{2}$ is dependent on $a, b$ and $f(t)$. 
Journal of Inequalities and Applications

The boundary value condition (1.4) implies that

$$
\begin{gathered}
\sum_{\ell=1}^{m-2} \alpha_{\ell}\left[\sum_{t_{i}<\eta_{\ell}} b_{i}+F(f)\left(\eta_{\ell}\right)\right]=0 \\
\int_{0}^{1} g(t)\left\{F\left\{\varphi^{-1}\left[t,(w(t))^{-1}\left(\rho_{2}+\sum_{t_{i}<t} b_{i}+F(f)(t)\right)\right]\right\}(t)+\sum_{t_{i}<t} a_{i}\right\} d t=0 .
\end{gathered}
$$

For any $\omega \in W$, we denote

$$
\Lambda_{\omega}\left(\rho_{2}\right)=\int_{0}^{1} g(t)\left\{F\left\{\varphi^{-1}\left[t,(w(t))^{-1}\left(\rho_{2}+\sum_{t_{i}<t} b_{i}+F(h)(t)\right)\right]\right\}(t)+\sum_{t_{i}<t} a_{i}\right\} d t
$$

Lemma 2.5. The function $\Lambda_{\omega}(\cdot)$ has the following properties.

(i) For any fixed $\omega \in W$, the equation

$$
\Lambda_{\omega}\left(\rho_{2}\right)=0
$$

has unique solution $\widetilde{\rho_{2}}(\omega) \in \mathbb{R}^{N}$.

(ii) The function $\widetilde{\rho_{2}}: W \rightarrow \mathbb{R}^{N}$, defined in (i), is continuous and sends bounded sets to bounded sets. Moreover, for any $\omega=(a, b, h) \in W$, we have

$$
\left|\widetilde{\rho_{2}}(\omega)\right| \leq 3 N\left[(2 N)^{p^{+}}\left(\sum_{i=1}^{k}\left|a_{i}\right|\right)^{p^{\sharp}-1}+\sum_{i=1}^{k}\left|b_{i}\right|+\|h\|_{L^{1}}\right]
$$

where

$$
M^{p^{*}-1}= \begin{cases}M^{p^{+}-1}, & M>1 \\ M^{p^{-}-1}, & M \leq 1\end{cases}
$$

Proof. (i) From Lemma 2.1, it is immediate that

$$
\left\langle\Lambda_{\omega}\left(x_{1}\right)-\Lambda_{\omega}\left(x_{2}\right), x_{1}-x_{2}\right\rangle>0, \quad \text { for } x_{1} \neq x_{2}, \forall x_{1}, x_{2} \in \mathbb{R}^{N},
$$

and hence, if (2.27) has a solution, then it is unique.

Set

$$
R_{0}=3 N\left[(2 N)^{p^{+}}\left(\sum_{i=1}^{k}\left|a_{i}\right|\right)^{p^{\#}-1}+\sum_{i=1}^{k}\left|b_{i}\right|+\|h\|_{L^{1}}\right]
$$


Suppose $\left|\rho_{2}\right|>R_{0}$, it is easy to see that there exists some $j_{0} \in\{1, \ldots, N\}$ such that, the absolute value of the $j_{0}$ th component $\rho_{2}^{j_{0}}$ of $\rho_{2}$ satisfies

$$
\left|\rho_{2}^{j_{0}}\right| \geq \frac{1}{N}\left|\rho_{2}\right|>\frac{1}{N} R_{0}=3\left[(2 N)^{p^{+}}\left(\sum_{i=1}^{k}\left|a_{i}\right|\right)^{p^{\#}-1}+\sum_{i=1}^{k}\left|b_{i}\right|+\|h\|_{L^{1}}\right] .
$$

Thus the $j_{0}$ th component of $\rho_{2}+\sum_{t_{i}<t} b_{i}+F(h)(t)$ keeps sign on $J$, then it is not hard to check that the $j_{0}$ th component of $\Lambda_{\omega}\left(\rho_{2}\right)$ keeps the same sign of $\rho_{2}^{j_{0}}$.

Thus $\Lambda_{\omega}\left(\rho_{2}\right) \neq 0$. Let us consider the equation

$$
\lambda \Lambda_{\omega}\left(\rho_{2}\right)+(1-\lambda) \rho_{2}=0, \quad \lambda \in[0,1] .
$$

According to the preceding discussion, all the solutions of (2.33) belong to $b\left(R_{0}+1\right)=$ $\left\{x \in \mathbb{R}^{N}|| x \mid<R_{0}+1\right\}$. Therefore

$$
d_{B}\left[\Lambda_{\omega}\left(\rho_{2}\right), b\left(R_{0}+1\right), 0\right]=d_{B}\left[I, b\left(R_{0}+1\right), 0\right] \neq 0,
$$

it means the existence of solutions of $\Lambda_{\omega}\left(\rho_{2}\right)=0$.

In this way, we define a function $\widetilde{\rho_{2}}(\omega): W \rightarrow \mathbb{R}^{N}$, which satisfies $\Lambda_{\omega}\left(\widetilde{\rho_{2}}(\omega)\right)=0$.

(ii) By the proof of (i), we also obtain $\widetilde{\rho_{2}}$ sends bounded sets to bounded sets, and

$$
\left|\widetilde{\rho_{2}}(\omega)\right| \leq 3 N\left[(2 N)^{p^{+}}\left(\sum_{i=1}^{k}\left|a_{i}\right|\right)^{p^{\#}-1}+\sum_{i=1}^{k}\left|b_{i}\right|+\|h\|_{L^{1}}\right] .
$$

It only remains to prove the continuity of $\widetilde{\rho_{2}}$. Let $\left\{\omega_{n}\right\}$ is a convergent sequence in $W$ and $\omega_{n} \rightarrow \omega$, as $n \rightarrow+\infty$. Since $\left\{\widetilde{\rho_{2}}\left(\omega_{n}\right)\right\}$ is a bounded sequence, it contains a convergent subsequence $\left\{\widetilde{\rho_{2}}\left(\omega_{n_{j}}\right)\right\}$. Suppose $\widetilde{\rho_{2}}\left(\omega_{n_{j}}\right) \rightarrow \rho_{2}^{0}$ as $j \rightarrow+\infty$. Since $\Lambda_{\omega_{n_{j}}}\left(\widetilde{\rho_{2}}\left(\omega_{n_{j}}\right)\right)=0$, letting $j \rightarrow+\infty$, we have $\Lambda_{\omega}\left(\rho_{2}^{0}\right)=0$, which together with (i) implies $\rho_{2}^{0}=\widetilde{\rho_{2}}(\omega)$, it means $\widetilde{\rho_{2}}$ is continuous. This completes the proof.

We define $\rho_{2}: \mathrm{PC}^{1} \rightarrow \mathbb{R}^{N}$ as

$$
\rho_{2}(u)=\widetilde{\rho_{2}}\left(A, B, N_{f}\right)(u),
$$

where $A=\left(A_{1}, \ldots, A_{k}\right), B=\left(B_{1}, \ldots, B_{k}\right)$.

It is clear that $\rho_{2}(\cdot)$ is continuous and sends bounded sets of $\mathrm{PC}^{1}$ to bounded sets of $\mathbb{R}^{N}$, and hence it is a compact continuous mapping. 
Let us define

$$
\begin{gathered}
P_{2}: \mathrm{PC}^{1} \longrightarrow \mathrm{PC}^{1}, u \longrightarrow u(0) ; \quad Q: L^{1} \longrightarrow L^{1}, \quad h \longrightarrow \sum_{\ell=1}^{m-2} \alpha_{\ell}\left[\sum_{t_{i}<\eta_{\ell}} b_{i}+F(h)\left(\eta_{\ell}\right)\right] \\
\Theta: L^{1} \longrightarrow L^{1}, h \longrightarrow h-\frac{1}{\sum_{\ell=1}^{m-2} \alpha_{\ell} \eta_{\ell}} Q h
\end{gathered}
$$

and $K_{(a, b)}^{*}: L^{1} \rightarrow \mathrm{PC}^{1}$ as

$$
K_{(a, b)}^{*}(h)(t)=F\left\{\varphi^{-1}\left[t,(w(t))^{-1}\left(\widetilde{\rho_{2}}(a, b, h)+\sum_{t_{i}<t} b_{i}+F(h)(t)\right)\right]\right\}(t), \quad \forall t \in J
$$

Similar to the proof of Lemma 2.3, we have the following lemma.

Lemma 2.6. The operator $\left(K_{(a, b)}^{*} \circ \Theta\right)(\cdot)$ is continuous and sends equiintegrable sets in $L^{1}$ to relatively compact sets in $P C^{1}$.

Denote

$$
\begin{gathered}
Q_{N_{f}}(u)=\sum_{\ell=1}^{m-2} \alpha_{\ell}\left[\sum_{t_{i}<\eta_{\ell}} B_{i}+F\left(N_{f}(u)\right)\left(\eta_{\ell}\right)\right], \\
\Theta_{f}(u)=N_{f}(u)-\frac{1}{\sum_{\ell=1}^{m-2} \alpha_{\ell} \eta_{\ell}} Q_{N_{f}}(u), \\
\rho_{2}(u)=\widetilde{\rho_{2}}\left(A, B, \Theta_{f}\right)(u), \\
K_{2}(u)(t)=F\left\{\varphi^{-1}\left[t,(w(t))^{-1}\left(\rho_{2}(u)+\sum_{t_{i}<t} B_{i}+F\left(\Theta_{f}(u)\right)(t)\right)\right]\right\}(t), \quad \forall t \in J .
\end{gathered}
$$

Lemma 2.7. Suppose $\sum_{\ell=1}^{m-2} \alpha_{\ell}=1$ and $\sigma=1$, then $u$ is a solution of (1.1)-(1.4) if and only if $u$ is a solution of the following abstract operator equation

$$
u=P_{2}(u)+\sum_{t_{i}<t} A_{i}+Q_{N_{f}}(u)+K_{2}(u)
$$

Proof. Suppose $u$ is a solution of (1.1)-(1.4), it is clear that $u$ is a solution of (2.40).

Conversely, if $u$ is a solution of (2.40), then (1.2) is satisfied and

$$
Q_{N_{f}}(u)=0 .
$$

Thus $\Theta_{f}(u)=N_{f}(u)$. 
From (2.40) and (2.41), we have

$$
\begin{gathered}
w(t) \varphi\left(t, u^{\prime}(t)\right)=\rho_{2}(u)+\sum_{t_{i}<t} B_{i}+F\left(\Theta_{f}(u)\right)(t), \quad t \in(0,1), t \neq t_{i} \\
\left(w(t) \varphi\left(t, u^{\prime}\right)\right)^{\prime}=N_{f}(u)(t), \quad t \in(0,1), t \neq t_{i} .
\end{gathered}
$$

According to (2.42), we get that (1.3) is satisfied. Since $Q_{N_{f}}(u)=0$, we have

$$
\lim _{t \rightarrow 0^{+}} w(t)\left|u^{\prime}\right|^{p(t)-2} u^{\prime}(t)=\sum_{\ell=1}^{m-2} \alpha_{\ell} \lim _{t \rightarrow \eta_{\ell}^{-}} w(t)\left|u^{\prime}\right|^{p(t)-2} u^{\prime}(t)
$$

It follows from the definition of $\rho_{2}$ that

$$
\int_{0}^{1} g(t)\left\{F\left\{\varphi^{-1}\left[t,(w(t))^{-1}\left(\rho_{2}(u)+\sum_{t_{i}<t} B_{i}+F\left(\Theta_{f}(u)\right)(t)\right)\right]\right\}(t)+\sum_{t_{i}<t} A_{i}\right\} d t=0
$$

then $u(0)=\int_{0}^{1} g(t) u(t) d t$.

Hence $u$ is a solutions of (1.1)-(1.4). This completes the proof.

\section{Existence of Solutions in the Case of Nonresonance}

In this section, we will apply Leray-Schauder's degree to deal with the existence of solutions and nonnegative solutions for system (1.1)-(1.4) at nonresonance.

When $f$ satisfies sub- $\left(p^{-}-1\right)$ growth condition, we have the following.

Theorem 3.1. Suppose $0 \leq \sum_{\ell=1}^{m-2} \alpha_{\ell}<1$ and $0 \leq \sigma<1, f$ satisfies sub- $\left(p^{-}-1\right)$ growth condition, and operators $A$ and $B$ satisfy the following condition

$$
\begin{array}{ll}
\sum_{i=1}^{k}\left|A_{i}(u, v)\right| \leq C_{1}(1+|u|+|v|)^{\left(q^{+}-1\right) /\left(p^{+}-1\right)}, & \\
\sum_{i=1}^{k}\left|B_{i}(u, v)\right| \leq C_{2}(1+|u|+|v|)^{q^{+}-1}, &
\end{array} \quad \forall(u, v) \in \mathbb{R}^{N} \times \mathbb{R}^{N},
$$

then problem (1.1)-(1.4) has at least one solution. 
Proof. First we consider the following problem:

$$
\begin{gathered}
-\Delta_{p(t)} u+\lambda N_{f}(u)(t)=0, \quad t \in(0,1), \quad t \neq t_{i}, \\
\lim _{t \rightarrow t_{i}^{+}} u(t)-\lim _{t \rightarrow t_{i}^{-}} u(t)=\lambda A_{i}\left(\lim _{t \rightarrow t_{i}^{-}} u(t), \lim _{t \rightarrow t_{i}^{-}}(w(t))^{1 /(p(t)-1)} u^{\prime}(t)\right), \quad i=1, \ldots, k, \\
\lim _{t \rightarrow t_{i}^{+}} w(t)\left|u^{\prime}\right|^{p(t)-2} u^{\prime}(t)-\lim _{t \rightarrow t_{i}^{-}} w(t)\left|u^{\prime}\right|^{p(t)-2} u^{\prime}(t) \\
=\lambda B_{i}\left(\lim _{t \rightarrow t_{i}^{-}} u(t), \lim _{t \rightarrow t_{i}^{-}}(w(t))^{1 /(p(t)-1)} u^{\prime}(t)\right), \quad i=1, \ldots, k, \\
\lim _{t \rightarrow 0^{+}} w(t)\left|u^{\prime}\right|^{p(t)-2} u^{\prime}(t)=\sum_{\ell=1}^{m-2} \alpha_{\ell} \lim _{t \rightarrow \eta_{\ell}^{-}} w(t)\left|u^{\prime}\right|^{p(t)-2} u^{\prime}(t), \quad u(0)=\int_{0}^{1} g(t) u(t) d t .
\end{gathered}
$$

Denote

$$
\begin{gathered}
\rho_{1, \lambda}(u)=\widetilde{\rho_{1}}\left(\lambda A, \lambda B, \lambda N_{f}\right)(u), \\
K_{1, \lambda}(u)=F\left\{\varphi^{-1}\left[t,(w(t))^{-1}\left(\rho_{1, \lambda}(u)+\lambda \sum_{t_{i}<t} B_{i}+F\left(\lambda N_{f}(u)\right)(t)\right)\right]\right\}, \\
P_{1, \lambda}(u)=\frac{1}{(1-\sigma)} \int_{0}^{1} g(t)\left[K_{1, \lambda}(u)(t)+\sum_{t_{i}<t} \lambda A_{i}\right] d t \\
\Psi_{f}(u, \lambda)=P_{1, \lambda}(u)+\lambda \sum_{t_{i}<t} A_{i}+K_{1, \lambda}(u),
\end{gathered}
$$

where $N_{f}(u)$ is defined in (2.11).

We know that $\left(S_{1}\right)$ has the same solution of the following operator equation when $\lambda=1$,

$$
u=\Psi_{f}(u, \lambda)
$$

It is easy to see that operator $\rho_{1, \lambda}$ is compact continuous for any $\lambda \in[0,1]$. It follows from Lemmas 2.2 and 2.3 that $\Psi_{f}(\cdot, \lambda)$ is compact continuous from $\mathrm{PC}^{1}$ to $\mathrm{PC}^{1}$ for any $\lambda \in$ $[0,1]$.

We claim that all the solutions of (3.3) are uniformly bounded for $\lambda \in[0,1]$. In fact, if it is false, we can find a sequence of solutions $\left\{\left(u_{n}, \lambda_{n}\right)\right\}$ for (3.3) such that $\left\|u_{n}\right\|_{1} \rightarrow+\infty$ as $n \rightarrow+\infty$, and $\left\|u_{n}\right\|_{1}>1$ for any $n=1,2, \ldots$

From Lemma 2.2, we have

$$
\left|\rho_{1, \lambda}(u)\right| \leq C_{3}\left[\sum_{i=1}^{k}\left|B_{i}\right|+\left\|N_{f}(u)\right\|_{L^{1}}\right] \leq C_{4}\left(1+\|u\|_{1}^{q^{+}-1}\right) .
$$


Thus

$$
\left|\rho_{1, \lambda}(u)+\sum_{t_{i}<t} \lambda B_{i}+F\left(\lambda N_{f}\right)\right| \leq\left|\rho_{1, \lambda}(u)\right|+\left|\sum_{t_{i}<t} B_{i}\right|+\left|F\left(N_{f}\right)\right| \leq C_{5}\left(1+\|u\|_{1}^{q^{+}-1}\right) .
$$

From $\left(S_{1}\right)$, we have

$$
w(t)\left|u_{n}^{\prime}(t)\right|^{p(t)-2} u_{n}^{\prime}(t)=\rho_{1, \lambda}\left(u_{n}\right)+\sum_{t_{i}<t} \lambda B_{i}+\int_{0}^{t} \lambda N_{f}\left(u_{n}\right)(s) d s, \quad \forall t \in J^{\prime} .
$$

It follows from (2.12) and Lemma 2.2 that

$$
w(t)\left|u_{n}^{\prime}(t)\right|^{p(t)-1} \leq\left|\rho_{1, \lambda}\left(u_{n}\right)\right|+\sum_{i=1}^{k}\left|B_{i}\right|+\int_{0}^{1}\left|N_{f}\left(u_{n}\right)(s)\right| d s \leq C_{6}+C_{7}\left\|u_{n}\right\|_{1}^{q^{+}-1}, \quad \forall t \in J^{\prime}
$$

Denote $\alpha=\left(q^{+}-1\right) /\left(p^{-}-1\right)$. The above inequality holds

$$
\left\|(w(t))^{1 /(p(t)-1)} u_{n}^{\prime}(t)\right\|_{0} \leq C_{8}\left\|u_{n}\right\|_{1}^{\alpha}, \quad n=1,2, \ldots
$$

It follows from (3.1) and (3.5) that

$$
\left|u_{n}(0)\right| \leq C_{9}\left\|u_{n}\right\|_{1}^{\alpha}, \quad \text { where } \alpha=\frac{q^{+}-1}{p^{-}-1} .
$$

For any $j=1, \ldots, N$, we have

$$
\begin{aligned}
\left|u_{n}^{j}(t)\right| & =\left|u_{n}^{j}(0)+\sum_{t_{i}<t} A_{i}^{j}+\int_{0}^{t}\left(u_{n}^{j}\right)^{\prime}(s) d s\right| \\
& \leq\left|u_{n}^{j}(0)\right|+\left|\sum_{t_{i}<t} A_{i}\right|+\left|\int_{0}^{t}(w(s))^{-1 /(p(s)-1)} \sup _{t \in(0,1)}\right|(w(t))^{1 /(p(t)-1)}\left(u_{n}^{j}\right)^{\prime}(t)|d s| \\
& \leq\left\|u_{n}\right\|_{1}^{\alpha}\left[C_{10}+C_{8} E\right]+\left|\sum_{t_{i}<t} A_{i}\right| \leq C_{11}\left\|u_{n}\right\|_{1}^{\alpha}, \quad \forall t \in J, n=1,2, \ldots,
\end{aligned}
$$

which implies that $\left|u_{n}^{j}\right|_{0} \leq C_{12}\left\|u_{n}\right\|_{1}^{\alpha}, j=1, \ldots, N ; n=1,2, \ldots$ Thus

$$
\left\|u_{n}\right\|_{0} \leq N C_{12}\left\|u_{n}\right\|_{1}^{\alpha}, \quad n=1,2, \ldots
$$

It follows from (3.8) and (3.11) that $\left\{\left\|u_{n}\right\|_{1}\right\}$ is uniformly bounded. 
Thus, we can choose a large enough $R_{0}>0$ such that all the solutions of (3.3) belong to $B\left(R_{0}\right)=\left\{u \in \mathrm{PC}^{1} \mid\|u\|_{1}<R_{0}\right\}$. Therefore the Leray-Schauder degree $d_{\mathrm{LS}}\left[I-\Psi_{f}(\cdot, \lambda), B\left(R_{0}\right), 0\right]$ is well defined for $\lambda \in[0,1]$, and

$$
d_{\mathrm{LS}}\left[I-\Psi_{f}(\cdot, 1), B\left(R_{0}\right), 0\right]=d_{\mathrm{LS}}\left[I-\Psi_{f}(\cdot, 0), B\left(R_{0}\right), 0\right]
$$

It is easy to see that $u$ is a solution of $u=\Psi_{f}(u, 0)$ if and only if $u$ is a solution of the following usual differential equation

$$
\begin{gathered}
-\Delta_{p(t)} u=0, \quad t \in(0,1), \\
\lim _{t \rightarrow 0^{+}} w(t)\left|u^{\prime}\right|^{p(t)-2} u^{\prime}(t)=\sum_{\ell=1}^{m-2} \alpha_{\ell} \lim _{t \rightarrow \eta_{\ell}} w(t)\left|u^{\prime}\right|^{p(t)-2} u^{\prime}(t), \quad u(0)=\int_{0}^{1} g(t) u(t) d t .
\end{gathered}
$$

Obviously, system $\left(S_{2}\right)$ possesses a unique solution $u_{0}$. Since $u_{0} \in B\left(R_{0}\right)$, we have

$$
d_{\mathrm{LS}}\left[I-\Psi_{f}(\cdot, 1), B\left(R_{0}\right), 0\right]=d_{\mathrm{LS}}\left[I-\Psi_{f}(\cdot, 0), B\left(R_{0}\right), 0\right] \neq 0,
$$

which implies that (1.1)-(1.4) has at least one solution. This completes the proof.

Theorem 3.2. Suppose $0 \leq \sum_{\ell=1}^{m-2} \alpha_{\ell}<1$ and $0 \leq \sigma<1, f$ satisfies sub- $\left(p^{-}-1\right)$ growth condition, and operators $A$ and $D$ satisfy the following

$$
\begin{array}{ll}
\sum_{i=1}^{k}\left|A_{i}(u, v)\right| \leq C_{1}(1+|u|+|v|)^{\left(q^{+}-1\right) /\left(p^{+}-1\right)}, & \\
\sum_{i=1}^{k}\left|D_{i}(u, v)\right| \leq C_{2}(1+|u|+|v|)^{\alpha_{i}^{+}}, &
\end{array} \quad \forall(u, v) \in \mathbb{R}^{N} \times \mathbb{R}^{N},
$$

where $\alpha_{i} \leq\left(q^{+}-1\right) /\left(p\left(r_{i}\right)-1\right)$, and $p\left(r_{i}\right)-1 \leq q^{+}-\alpha_{i}, i=1, \ldots, k$, then problem (1.1) with (1.2), (1.4), and (1.8) has at least one solution.

Proof. Obviously,

$$
B_{i}(u, v)=\varphi\left(r_{i}, v+D_{i}(u, v)\right)-\varphi\left(r_{i}, v\right)
$$

From Theorem 3.1, it suffices to show that

$$
\sum_{i=1}^{k}\left|B_{i}(u, v)\right| \leq C_{2}(1+|u|+|v|)^{q^{+}-1}, \quad \forall(u, v) \in \mathbb{R}^{N} \times \mathbb{R}^{N}
$$


(a) Suppose $|v| \leq M^{*}\left|D_{i}(u, v)\right|$, where $M^{*}$ is a large enough positive constant. From the definition of $D$, we have

$$
\left|B_{i}(u, v)\right| \leq C_{1}\left|D_{i}(u, v)\right|^{p\left(r_{i}\right)-1} \leq C_{2}(1+|u|+|v|)^{\alpha_{i}\left(p\left(r_{i}\right)-1\right)} .
$$

Since $\alpha_{i}<\left(q^{+}-1\right) /\left(p\left(r_{i}\right)-1\right)$, we have $\alpha_{i}\left(p\left(r_{i}\right)-1\right) \leq q^{+}-1$. Thus (3.16) is valid.

(b) Suppose $|v|>M^{*}\left|D_{i}(u, v)\right|$, we have

$$
\left|B_{i}(u, v)\right| \leq C_{3}|v|^{p\left(r_{i}\right)-1} \frac{\left|D_{i}(u, v)\right|}{|v|}=C_{4}|v|^{p\left(r_{i}\right)-2}\left|D_{i}(u, v)\right| .
$$

There are two cases.

Case $1\left(p\left(r_{i}\right)-1 \geq 1\right)$. Since $p\left(r_{i}\right)-1 \leq q^{+}-\alpha_{i}$, we have $p\left(r_{i}\right)-2+\alpha_{i} \leq q^{+}-1$, and then

$$
\left|B_{i}(u, v)\right| \leq C_{5}|v|^{p\left(r_{i}\right)-2}\left|D_{i}(u, v)\right| \leq C_{6}(1+|u|+|v|)^{p\left(r_{i}\right)-2+\alpha_{i}} \leq C_{6}(1+|u|+|v|)^{q^{+}-1} .
$$

Thus (3.16) is valid.

Case $2\left(p\left(r_{i}\right)-1<1\right)$. Since $\alpha_{i}<\left(q^{+}-1\right) /\left(p\left(r_{i}\right)-1\right)$, we have $\alpha_{i}\left(p\left(r_{i}\right)-1\right) \leq q^{+}-1$, and

$$
\left|B_{i}(u, v)\right| \leq C_{7}|v|^{p\left(r_{i}\right)-2}\left|D_{i}(u, v)\right| \leq C_{8}\left|D_{i}(u, v)\right|^{p\left(r_{i}\right)-1} \leq C_{9}(1+|u|+|v|)^{\alpha_{i}\left(p\left(r_{i}\right)-1\right)}
$$

Thus (3.16) is valid. Thus problem (1.1) with (1.2), (1.4), and (1.8) has at least one solution. This completes the proof.

Let us consider

$$
-\Delta_{p(t)} u+\phi\left(t, u,(w(t))^{1 /(p(t)-1)} u^{\prime}, S(u), T(u), \delta\right)=0, \quad t \in(0,1), t \neq t_{i}
$$

where $\delta$ is a parameter, and

$$
\begin{aligned}
& \phi\left(t, u,(w(t))^{1 /(p(t)-1)} u^{\prime}, S(u), T(u), \delta\right) \\
& \quad=f\left(t, u,(w(t))^{1 /(p(t)-1)} u^{\prime}, S(u), T(u)\right)+\delta h\left(t, u,(w(t))^{1 /(p(t)-1)} u^{\prime}, S(u), T(u)\right)
\end{aligned}
$$

where $h, f: J \times \mathbb{R}^{N} \times \mathbb{R}^{N} \times \mathbb{R}^{N} \times \mathbb{R}^{N} \rightarrow \mathbb{R}^{N}$ are Caratheodory. 
We have the following.

Theorem 3.3. Suppose $0 \leq \sum_{\ell=1}^{m-2} \alpha_{\ell}<1$ and $0 \leq \sigma<1, f$ satisfies sub- $\left(p^{-}-1\right)$ growth condition, and we assume that

$$
\begin{array}{ll}
\sum_{i=1}^{k}\left|A_{i}(u, v)\right| \leq C_{1}(1+|u|+|v|)^{\left(q^{+}-1\right) /\left(p^{+}-1\right)}, & \\
\sum_{i=1}^{k}\left|B_{i}(u, v)\right| \leq C_{2}(1+|u|+|v|)^{q^{+}-1}, &
\end{array}
$$

then problem (3.21) with (1.2)-(1.4) has at least one solution when the parameter $\delta$ is small enough.

Proof. Denote

$$
\begin{aligned}
& \phi_{\lambda}\left(t, u,(w(t))^{1 /(p(t)-1)} u^{\prime}, S(u), T(u), \delta\right) \\
& \quad=f\left(t, u,(w(t))^{1 /(p(t)-1)} u^{\prime}, S(u), T(u)\right)+\lambda \delta h\left(t, u,(w(t))^{1 /(p(t)-1)} u^{\prime}, S(u), T(u)\right) .
\end{aligned}
$$

We consider the existence of solutions of the following equation with (1.2)-(1.4)

$$
-\Delta_{p(t)} u+\phi_{\lambda}\left(t, u,(w(t))^{1 /(p(t)-1)} u^{\prime}, S(u), T(u), \delta\right)=0, \quad t \in(0,1), t \neq t_{i} .
$$

Denote

$$
\begin{gathered}
\rho_{1, \lambda}^{\#}(u, \delta)=\widetilde{\rho_{1}}\left(A, B, N_{\phi_{\lambda}}\right)(u), \\
K_{1, \lambda}^{\#}(u, \delta)=F\left\{\varphi^{-1}\left[t,(w(t))^{-1}\left(\rho_{1, \lambda}^{\#}(u, \delta)+\sum_{t_{i}<t} B_{i}+F\left(N_{\phi_{\lambda}}(u)\right)(t)\right)\right]\right\}, \\
P_{1, \lambda}^{\#}(u, \delta)=\frac{1}{(1-\sigma)} \int_{0}^{1} g(t)\left[K_{1, \lambda}^{\#}(u, \delta)(t)+\sum_{t_{i}<t} A_{i}\right] d t, \\
\Phi_{\delta}(u, \lambda)=P_{1, \lambda}^{\#}(u, \delta)+\sum_{t_{i}<t} A_{i}+K_{1, \lambda}^{\#}(u, \delta),
\end{gathered}
$$

where $N_{\phi_{\lambda}}(u)$ is defined in (2.11).

We know that (3.25) with (1.2)-(1.4) has the same solution of $u=\Phi_{\delta}(u, \lambda)$.

Obviously, $\phi_{0}=f$. So $\Phi_{\delta}(u, 0)=\Psi_{f}(u, 1)$. As in the proof of Theorem 3.1, we know that all the solutions of $u=\Phi_{\delta}(u, 0)$ are uniformly bounded, then there exists a large enough $R_{0}>0$ such that all the solutions of $u=\Phi_{\delta}(u, 0)$ belong to $B\left(R_{0}\right)=\left\{u \in \mathrm{PC}^{1} \mid\|u\|_{1}<R_{0}\right\}$. Since $\Phi_{\delta}(\cdot, 0)$ is compact continuous from $\mathrm{PC}^{1}$ to $P C^{1}$, we have

$$
\inf _{u \in \partial B\left(R_{0}\right)}\left\|u-\Phi_{\delta}(u, 0)\right\|_{1}>0
$$


Since $f, h$ are Caratheodory, we have

$$
\begin{array}{r}
\left\|F\left(N_{\phi_{\lambda}}(u)\right)-F\left(N_{\phi_{0}}(u)\right)\right\|_{0} \longrightarrow 0 \quad \text { for }(u, \lambda) \in \overline{B\left(R_{0}\right)} \times[0,1] \text { uniformly, as } \delta \longrightarrow 0, \\
\left|\rho_{1, \lambda}^{\#}(u, \delta)-\rho_{1,0}^{\#}(u, \delta)\right| \longrightarrow 0 \quad \text { for }(u, \lambda) \in \overline{B\left(R_{0}\right)} \times[0,1] \text { uniformly, as } \delta \longrightarrow 0, \\
\left\|K_{1, \lambda}^{\#}(u, \delta)-K_{1,0}^{\#}(u, \delta)\right\|_{1} \longrightarrow 0 \quad \text { for }(u, \lambda) \in \overline{B\left(R_{0}\right)} \times[0,1] \text { uniformly, as } \delta \longrightarrow 0, \\
\left|\rho_{1, \lambda}^{\#}(u, \delta)-P_{1,0}^{\#}(u, \delta)\right| \longrightarrow 0 \quad \text { for }(u, \lambda) \in \overline{B\left(R_{0}\right)} \times[0,1] \text { uniformly, as } \delta \longrightarrow 0 .
\end{array}
$$

Thus

$\left\|\Phi_{\delta}(u, \lambda)-\Phi_{0}(u, \lambda)\right\|_{1} \longrightarrow 0$ for $(u, \lambda) \in \overline{B\left(R_{0}\right)} \times[0,1]$ uniformly, as $\delta \longrightarrow 0$,

Obviously, $\Phi_{0}(u, \lambda)=\Phi_{\delta}(u, 0)=\Phi_{0}(u, 0)$. We obtain

$\left\|\Phi_{\delta}(u, \lambda)-\Phi_{\delta}(u, 0)\right\|_{1} \longrightarrow 0$ for $(u, \lambda) \in \overline{B\left(R_{0}\right)} \times[0,1]$ uniformly, as $\delta \longrightarrow 0$

Thus, when $\delta$ is small enough, we can conclude that

$$
\begin{aligned}
& \quad \inf _{(u, \lambda) \in \partial B\left(R_{0}\right) \times[0,1]}\left\|u-\Phi_{\delta}(u, \lambda)\right\|_{1} \\
& \quad \geq \inf _{u \in \partial B\left(R_{0}\right)}\left\|u-\Phi_{\delta}(u, 0)\right\|_{1}-\sup _{(u, \lambda) \in \overline{B\left(R_{0}\right) \times[0,1]}}\left\|\Phi_{\delta}(u, 0)-\Phi_{\delta}(u, \lambda)\right\|_{1}>0 .
\end{aligned}
$$

Thus $u=\Phi_{\delta}(u, \lambda)$ has no solution on $\partial B\left(R_{0}\right)$ for any $\lambda \in[0,1]$, when $\delta$ is small enough. It means that the Leray-Schauder degree $d_{\mathrm{LS}}\left[I-\Phi_{\delta}(\cdot, \lambda), B\left(R_{0}\right), 0\right]$ is well defined for any $\lambda \in[0,1]$, and

$$
d_{\mathrm{LS}}\left[I-\Phi_{\delta}(u, \lambda), B\left(R_{0}\right), 0\right]=d_{\mathrm{LS}}\left[I-\Phi_{\delta}(u, 0), B\left(R_{0}\right), 0\right]
$$

Since $\Phi_{\delta}(u, 0)=\Psi_{f}(u, 1)$, from the proof of Theorem 3.1, we can see that the right hand side is nonzero. Thus (3.21) with (1.2)-(1.4) has at least one solution. This completes the proof.

Theorem 3.4. Suppose $0 \leq \sum_{\ell=1}^{m-2} \alpha_{\ell}<1$ and $0 \leq \sigma<1, f$ satisfies sub- $\left(p^{-}-1\right)$ growth condition, and we assume that

$$
\begin{aligned}
& \sum_{i=1}^{k}\left|A_{i}(u, v)\right| \leq C_{1}(1+|u|+|v|)^{\left(q^{+}-1\right) /\left(p^{+}-1\right)}, \\
& \sum_{i=1}^{k}\left|D_{i}(u, v)\right| \leq C_{2}(1+|u|+|v|)^{\alpha_{i}^{+}},
\end{aligned}
$$


where $\alpha_{i} \leq\left(q^{+}-1\right) /\left(p\left(r_{i}\right)-1\right)$, and $p\left(r_{i}\right)-1 \leq q^{+}-\alpha_{i}, i=1, \ldots, k$, then problem (3.21) with (1.2), (1.4), and (1.8) has at least one solution when the parameter $\delta$ is small enough.

Proof. As it is similar to the proof of Theorems 3.2 and 3.3, we omit it here.

In the following, we will consider the existence of nonnegative solutions. For any $x=$ $\left(x^{1}, \ldots, x^{N}\right) \in \mathbb{R}^{N}$, the notation $x \geq 0$ means $x^{j} \geq 0$ for any $j=1, \ldots, N$.

Theorem 3.5. Suppose $0 \leq \sum_{\ell=1}^{m-2} \alpha_{\ell}<1,0 \leq \sigma<1$, we also assume

$\left(1^{0}\right) f(t, x, y, s, z) \geq 0$, for all $(t, x, y, s, z) \in J \times \mathbb{R}^{N} \times \mathbb{R}^{N} \times \mathbb{R}^{N} \times \mathbb{R}^{N}$;

$\left(2^{0}\right)$ for any $i=1, \ldots, k, B_{i}(u, v) \geq 0$, for all $(u, v) \in \mathbb{R}^{N} \times \mathbb{R}^{N}$.

Then every solution of (1.1)-(1.4) is nonnegative.

Proof. Let $u$ be a solution of (1.1)-(1.4), integrating (1.1) from 0 to $t$, we have

$$
w(t) \varphi\left(t, u^{\prime}(t)\right)=\rho_{1}(u)+\sum_{t_{i}<t} B_{i}+F\left(N_{f}(u)\right)(t), \quad \forall t \in(0,1), t \neq t_{1}, \ldots, t_{k},
$$

where $\rho_{1}=w(0) \varphi\left(0, u^{\prime}(0)\right)$. The boundary value condition holds

$$
\rho_{1}=\frac{\left\{\sum_{\ell=1}^{m-2} \alpha_{\ell}\left[\sum_{t_{i}<\eta_{\ell}} B_{i}+F\left(N_{f}(u)\right)\left(\eta_{\ell}\right)\right]\right\}}{1-\sum_{\ell=1}^{m-2} \alpha_{\ell}} .
$$

Conditions $\left(1^{0}\right)-\left(2^{0}\right)$ mean $\rho_{1}(u) \geq 0$. Obviously, for any for all $t \in J^{\prime}$, we have

$$
w(t) \varphi\left(t, u^{\prime}(t)\right)=\rho_{1}(u)+\sum_{t_{i}<t} B_{i}+F\left(N_{f}(u)\right)(t) \geq 0 .
$$

It follows from conditions $\left(1^{0}\right)-\left(2^{0}\right)$ and (3.36) that $u(t)$ is increasing on $J$, namely $u\left(t^{\prime}\right)-u\left(t^{\prime \prime}\right) \geq 0$, for all $t^{\prime}, t^{\prime \prime} \in J$ with $t^{\prime} \geq t^{\prime \prime}$. Thus the boundary value condition holds $u(0)=\int_{0}^{1} g(t) u(t) d t \geq \int_{0}^{1} g(t) u(0) d t=\sigma u(0)$, then $u(0) \geq 0$.

Since $u(t)$ is increasing and $u(0) \geq 0$, we have $u(t) \geq 0$, for all $t \in J$.

Thus every solution of (1.1)-(1.4) is nonnegative. The proof is completed.

Corollary 3.6. Under the conditions of Theorem 3.1, we also assume

$\left(1^{0}\right) f(t, x, y, s, z) \geq 0$, for all $(t, x, y, s, z) \in J \times \mathbb{R}^{N} \times \mathbb{R}^{N} \times \mathbb{R}^{N} \times \mathbb{R}^{N}$ with $x, s, z \geq 0$;

$\left(2^{0}\right)$ for any $i=1, \ldots, k, B_{i}(u, v) \geq 0$, for all $(u, v) \in \mathbb{R}^{N} \times \mathbb{R}^{N}$ with $u \geq 0$;

$\left(3^{0}\right)$ for any $t \in[0,1]$ and $s \in[0,1], k_{*}(t, s) \geq 0, h_{*}(t, s) \geq 0$.

Then (1.1)-(1.4) has a nonnegative solution.

Proof. Define $M(u)=\left(M_{*}\left(u^{1}\right), \ldots, M_{*}\left(u^{N}\right)\right)$, where

$$
M_{*}(u)= \begin{cases}u, & u \geq 0, \\ 0, & u<0 .\end{cases}
$$


Denote

$$
\tilde{f}(t, u, v, S(u), T(u))=f(t, M(u), v, S(M(u)), T(M(u))), \quad \forall(t, u, v) \in J \times \mathbb{R}^{N} \times \mathbb{R}^{N},
$$

then $\tilde{f}(t, u, v, S(u), T(u))$ satisfies Caratheodory condition, and $\tilde{f}(t, u, v, S(u), T(u)) \geq 0$ for any $(t, u, v) \in J \times \mathbb{R}^{N} \times \mathbb{R}^{N}$.

For any $i=1, \ldots, k$, we denote

$$
\tilde{A}_{i}(u, v)=A_{i}(M(u), v), \quad \tilde{B}_{i}(u, v)=B_{i}(M(u), v), \quad \forall(u, v) \in \mathbb{R}^{N} \times \mathbb{R}^{N},
$$

then $\widetilde{A}_{i}$ and $\widetilde{B}_{i}$ are continuous, and satisfy

$$
\widetilde{B}_{i}(u, v) \geq 0, \quad \forall(u, v) \in \mathbb{R}^{N} \times \mathbb{R}^{N} \text { for any } i=1, \ldots, k .
$$

It is not hard to check that

$\left(2^{0}\right)^{\prime} \lim _{|u|+|v| \rightarrow+\infty}\left(\tilde{f}(t, u, v, S(u), T(u)) /(|u|+|v|)^{q(t)-1}\right)=0$, for $t \in J$ uniformly, where $q(t) \in C(J, \mathbb{R})$, and $1<q^{-} \leq q^{+}<p^{-} ;$

$\left(3^{0}\right)^{\prime} \sum_{i=1}^{k}\left|\tilde{A}_{i}(u, v)\right| \leq C_{1}(1+|u|+|v|)^{\left(q^{+}-1\right) /\left(p^{+}-1\right)}$, for all $(u, v) \in \mathbb{R}^{N} \times \mathbb{R}^{N}$;

$\left(4^{0}\right)^{\prime} \sum_{i=1}^{k}\left|\widetilde{B}_{i}(u, v)\right| \leq C_{2}(1+|u|+|v|)^{q^{+}-1}$, for all $(u, v) \in \mathbb{R}^{N} \times \mathbb{R}^{N}$.

Let us consider

$$
\begin{gathered}
-\Delta_{p(t)} u+\tilde{f}\left(t, u,(w(t))^{1 /(p(t)-1)} u^{\prime}, S(u), T(u)\right)=0, \quad t \in J^{\prime}, \\
\lim _{t \rightarrow t_{i}^{+}} u(t)-\lim _{t \rightarrow t_{i}^{-}} u\left(t_{i}\right)=\tilde{A}_{i}\left(\lim _{t \rightarrow t_{i}^{-}} u(t), \lim _{t \rightarrow t_{i}^{-}}(w(t))^{1 /(p(t)-1)} u^{\prime}(t)\right), \quad i=1, \ldots, k, \\
\lim _{t \rightarrow t_{i}^{+}} w(t) \varphi\left(t, u^{\prime}(t)\right)-\lim _{t \rightarrow t_{i}^{-}} w(t) \varphi\left(t, u^{\prime}(t)\right) \\
=\tilde{B}_{i}\left(\lim _{t \rightarrow t_{i}^{-}} u(t), \lim _{t \rightarrow t_{i}^{-}}(w(t))^{1 /(p(t)-1)} u^{\prime}(t)\right), \quad i=1, \ldots, k, \\
\lim _{t \rightarrow 0^{+}} w(t)\left|u^{\prime}\right|^{p(t)-2} u^{\prime}(t)=\sum_{\ell=1}^{m-2} \alpha_{\ell} \lim _{t \rightarrow \eta_{e}^{-}} w(t)\left|u^{\prime}\right|^{p(t)-2} u^{\prime}(t), \quad u(0)=\int_{0}^{1} g(t) u(t) d t .
\end{gathered}
$$

It follows from Theorems 3.1 and 3.5 that (3.41) have a nonnegative solution $u$. Since $u \geq 0$, we have $M(u)=u$. Thus $u$ is a nonnegative solution of (1.1)-(1.4). This completes the proof.

\section{Existence of Solutions in the Case of Resonance}

In the following, we will consider the existence of solutions for system (1.1)-(1.4) at resonance. 
Journal of Inequalities and Applications

Theorem 4.1. Suppose $\sum_{\ell=1}^{m-2} \alpha_{\ell}=1$ and $\sigma=1, \Omega$ is an open bounded set in $P C^{1}$ such that the following conditions hold.

$\left(1^{0}\right)$ For each $\lambda \in(0,1)$ the problem

$$
\begin{gathered}
-\Delta_{p(t)} u+\lambda N_{f}(u)(t)=0, \quad t \in(0,1), \quad t \neq t_{i}, \\
\lim _{t \rightarrow t_{i}^{+}} u(t)-\lim _{t \rightarrow t_{i}^{-}} u(t)=\lambda A_{i}\left(\lim _{t \rightarrow t_{i}^{-}} u(t), \lim _{t \rightarrow t_{i}^{-}}(w(t))^{1 /(p(t)-1)} u^{\prime}(t)\right), \quad i=1, \ldots, k, \\
\lim _{t \rightarrow t_{i}^{+}} w(t)\left|u^{\prime}\right|^{p(t)-2} u^{\prime}(t)-\lim _{t \rightarrow t_{i}^{-}} w(t)\left|u^{\prime}\right|^{p(t)-2} u^{\prime}(t) \\
=\lambda B_{i}\left(\lim _{t \rightarrow t_{i}^{-}} u(t), \lim _{t \rightarrow t_{i}^{-}}(w(t))^{1 /(p(t)-1)} u^{\prime}(t)\right), \quad i=1, \ldots, k, \\
\lim _{t \rightarrow 0^{+}} w(t)\left|u^{\prime}\right|^{p(t)-2} u^{\prime}(t)=\sum_{\ell=1}^{m-2} \alpha_{\ell} \lim _{t \rightarrow \eta_{e}^{-}} w(t)\left|u^{\prime}\right|^{p(t)-2} u^{\prime}(t), \quad u(0)=\int_{0}^{1} g(t) u(t) d t .
\end{gathered}
$$

has no solution on $\partial \Omega$.

$\left(2^{0}\right)$ The equation

$$
\omega(l):=\left\{\sum_{\ell=1}^{m-2} \alpha_{\ell}\left[\sum_{t_{i}<\eta_{\ell}} B_{i}(l, 0)+\int_{0}^{\eta_{\ell}} f(t, l, 0, S(l), T(l)) d t\right]\right\}=0
$$

has no solution on $\partial \Omega \cap \mathbb{R}^{N}$.

$\left(3^{0}\right)$ The Brouwer degree $d_{B}\left[\omega, \Omega \cap \mathbb{R}^{N}, 0\right] \neq 0$.

Then problem (1.1)-(1.4) have a solution on $\bar{\Omega}$.

Proof. Let us consider the following impulsive equation

$$
\begin{gathered}
-\Delta_{p(t)} u+\lambda N_{f}(u)(t)+\frac{(1-\lambda)\left[Q_{N_{f}}(u)\right]}{\sum_{\ell=1}^{m-2} \alpha_{\ell} \eta_{\ell}}=0, \quad t \in(0,1), \quad t \neq t_{i}, \\
\lim _{t \rightarrow t_{i}^{+}} u(t)-\lim _{t \rightarrow t_{i}^{-}} u(t)=\lambda A_{i}\left(\lim _{t \rightarrow t_{i}^{-}} u(t), \lim _{t \rightarrow t_{i}^{-}}(w(t))^{1 /(p(t)-1)} u^{\prime}(t)\right), \quad i=1, \ldots, k, \\
\lim _{t \rightarrow t_{i}^{+}} w(t)\left|u^{\prime}\right|^{p(t)-2} u^{\prime}(t)-\lim _{t \rightarrow t_{i}^{-}} w(t)\left|u^{\prime}\right|^{p(t)-2} u^{\prime}(t) \\
=\lambda B_{i}\left(\lim _{t \rightarrow t_{i}^{-}} u(t), \lim _{t \rightarrow t_{i}^{-}}(w(t))^{1 /(p(t)-1)} u^{\prime}(t)\right), \quad i=1, \ldots, k, \\
\lim _{t \rightarrow 0^{+}} w(t)\left|u^{\prime}\right|^{p(t)-2} u^{\prime}(t)=\sum_{\ell=1}^{m-2} \alpha_{\ell} \lim _{t \rightarrow \eta_{\ell}^{-}} w(t)\left|u^{\prime}\right|^{p(t)-2} u^{\prime}(t), \quad u(0)=\int_{0}^{1} g(t) u(t) d t .
\end{gathered}
$$


For any $\lambda \in(0,1]$, if $u$ is a solution to (4.1) or $u$ is a solution to (4.3), we have necessarily

$$
Q_{N_{f}}(u)=0 .
$$

It means that (4.1) and (4.3) have the same solutions for $\lambda \in(0,1]$.

We denote $N(\cdot, \cdot): \mathrm{PC}^{1} \times[0,1] \rightarrow L^{1}$ defined by

$$
N(u, \lambda)=\lambda N_{f}(u)+\frac{(1-\lambda)\left[Q_{N_{f}}(u)\right]}{\sum_{\ell=1}^{m-2} \alpha_{\ell} \eta_{\ell}},
$$

where $N_{f}(u)$ is defined by (2.11). Denote

$$
\begin{gathered}
Q_{\lambda}: L^{1} \longrightarrow L^{1}, u \longrightarrow \sum_{\ell=1}^{m-2} \alpha_{\ell}\left[\lambda \sum_{t_{i}<\eta_{\ell}} B_{i}+F(N(u, \lambda))\left(\eta_{\ell}\right)\right], \\
\Theta_{\lambda}: L^{1} \longrightarrow L^{1}, u \longrightarrow N(u, \lambda)-\frac{Q_{\lambda}(u)}{\sum_{\ell=1}^{m-2} \alpha_{\ell} \eta_{\ell}}, \\
\rho_{2, \lambda}(u)=\widetilde{\rho_{2}}\left(\lambda A, \lambda B, \Theta_{\lambda}\right), \\
K_{2, \lambda}(u)(t)=F\left\{\varphi^{-1}\left[t,(w(t))^{-1}\left(\rho_{2, \lambda}(u)+\lambda \sum_{t_{i}<t} B_{i}+F\left(\Theta_{\lambda}(u)\right)(t)\right)\right]\right\}(t), \quad \forall t \in J .
\end{gathered}
$$

Set

$$
\Psi_{f}^{*}(u, \lambda)=P_{2}(u)+\lambda \sum_{t_{i}<t} A_{i}+Q_{\lambda}(u)+K_{2, \lambda}(u)
$$

then the fixed point of $\Psi_{f}^{*}(u, 1)$ is a solution for (1.1)-(1.4). Also problem (4.3) can be rewritten in the equivalent form

$$
u=\Psi_{f}^{*}(u, \lambda)
$$

Since $f$ is Caratheodory, it is easy to see that $N(\cdot, \cdot)$ is continuous and sends bounded sets into equiintegrable sets. It is easy to see that $P_{2}$ is compact continuous. From Lemma 2.6, we can conclude that $\Psi_{f}^{*}(u, \lambda)$ is continuous and compact for any $\lambda \in[0,1]$. We assume that (4.8) does not have a solution on $\partial \Omega$ for $\lambda=1$, otherwise we complete the proof. Now from hypothesis $\left(1^{0}\right)$ it follows that $(4.8)$ has no solutions for $(u, \lambda) \in \partial \Omega \times(0,1]$. For $\lambda=0,(4.3)$ is equivalent to the following usual problem

$$
\begin{gathered}
-\Delta_{p(t)} u+\frac{\left[Q N_{f}(u)\right]}{\sum_{\ell=1}^{m-2} \alpha_{\ell} \eta_{\ell}}=0, \quad t \in(0,1), \\
\lim _{t \rightarrow 0^{+}} w(t)\left|u^{\prime}\right|^{p(t)-2} u^{\prime}(t)=\sum_{\ell=1}^{m-2} \alpha_{\ell} \lim _{t \rightarrow \eta_{\ell}^{-}} w(t)\left|u^{\prime}\right|^{p(t)-2} u^{\prime}(t), \quad u(0)=\int_{0}^{1} g(t) u(t) d t .
\end{gathered}
$$


If $u$ is a solution to this problem, we must have

$$
0=\sum_{\ell=1}^{m-2} \alpha_{\ell}\left[\sum_{t_{i}<\eta \ell} B_{i}\left(\lim _{t \rightarrow t_{i}^{-}} u(t), \lim _{t \rightarrow t_{i}^{-}}(w(t))^{1 /(p(t)-1)} u^{\prime}(t)\right)+\int_{0}^{\eta_{\ell}} N_{f}(u) d t\right] .
$$

As this problem is a usual differential equation, we have

$$
w(t)\left|u^{\prime}\right|^{p(t)-2} u^{\prime} \equiv \rho_{2}
$$

where $\rho_{2} \in \mathbb{R}^{N}$ is a constant. Therefore $\left(u^{i}\right)^{\prime}$ keeps the same sign of $\rho_{2}^{i}$. From $u(0)=$ $\int_{0}^{1} g(t) u(t) d t$, we have $\int_{0}^{1} g(t)[u(0)-u(t)] d t=0$. From the continuity of $u$, there exist $t_{i} \in(0,1)$, such that $\left(u^{i}\right)^{\prime}\left(t_{i}\right)=0, i=1, \ldots, N$. Hence $u^{\prime} \equiv 0$, it holds $u \equiv d$, a constant. Thus (4.10) holds

$$
\sum_{\ell=1}^{m-2} \alpha_{\ell}\left[\sum_{t_{i}<\eta_{\ell}} B_{i}(l, 0)+\int_{0}^{\eta_{\ell}} f(t, l, 0, S(l), T(l)) d t\right]=0,
$$

which together with hypothesis $\left(2^{0}\right)$ implies that $u=d \notin \partial \Omega$. Thus we have proved that (4.8) has no solution $(u, \lambda)$ on $\partial \Omega \times[0,1]$. Therefore the Leray-Schauder degree $d_{\mathrm{LS}}[I-$ $\left.\Psi_{f}^{*}(\cdot, \lambda), \Omega, 0\right]$ is well defined for $\lambda \in[0,1]$, and from the homotopy invariant property of that degree we have

$$
d_{\mathrm{LS}}\left[I-\Psi_{f}^{*}(\cdot, 1), \Omega, 0\right]=d_{\mathrm{LS}}\left[I-\Psi_{f}^{*}(\cdot, 0), \Omega, 0\right]
$$

Now it is clear that the problem

$$
u=\Psi_{f}^{*}(u, 1)
$$

is equivalent to problem (1.1)-(1.4), and (4.13) tells us that problem (4.14) will have a solution if we can show that

$$
d_{\mathrm{LS}}\left[I-\Psi_{f}^{*}(\cdot, 0), \Omega, 0\right] \neq 0
$$

It is not hard to check that $K_{2,0}(\cdot) \equiv 0$. Thus

$$
\begin{gathered}
\Psi_{f}^{*}(u, 0)=P_{2} u+Q N_{f}(u)+K_{2,0}(u)=P_{2} u+Q N_{f}(u), \\
u-\Psi_{f}^{*}(u, 0)=u-P_{2} u-Q N_{f}(u)=-Q N_{f}(u), \quad \text { on } \bar{\Omega} .
\end{gathered}
$$

By the properties of the Leray-Schauder degree, we have

$$
d_{\mathrm{LS}}\left[I-\Psi_{f}^{*}(\cdot, 0), \Omega, 0\right]=(-1)^{N} d_{B}\left[\omega, \Omega \cap \mathbb{R}^{N}, 0\right],
$$


where the function $\omega$ is defined in (4.2) and $d_{B}$ denotes the Brouwer degree. By hypothesis $\left(3^{0}\right)$, this last degree is different from zero. This completes the proof.

Our next theorem is a consequence of Theorem 4.1. As an application of Theorem 4.1, let us consider the following system

$$
-\Delta_{p(t)} u+\gamma\left(t, u,(w(t))^{1 /(p(t)-1)} u^{\prime}, S(u), T(u)\right)+e\left(t, u,(w(t))^{1 /(p(t)-1)} u^{\prime}, S(u), T(u)\right)=0, \quad t \in J^{\prime},
$$

with (1.2), (1.3), and (1.4), where $e: J \times \mathbb{R}^{N} \times \mathbb{R}^{N} \times \mathbb{R}^{N} \times \mathbb{R}^{N} \rightarrow \mathbb{R}^{N}$ is Caratheodory, $\gamma=$ $\left(\gamma^{1}, \ldots, \gamma^{N}\right): J \times \mathbb{R}^{N} \times \mathbb{R}^{N} \times \mathbb{R}^{N} \times \mathbb{R}^{N} \rightarrow \mathbb{R}^{N}$ is continuous, and for any fixed $y_{0} \in \mathbb{R}^{N}, y_{0}^{i} \neq 0$ holds $\gamma^{i}\left(t, y_{0}, 0, S\left(y_{0}\right), T\left(y_{0}\right)\right) \neq 0$, for all $t \in J, i=1, \ldots, N$.

Theorem 4.2. Suppose that the following conditions hold

$\left(1^{0}\right) \gamma(t, k x, k y, k s, k z)=k^{q(t)-1} \gamma(t, x, y, s, z)$ for all $k>0$ and all $(t, x, y, s, z) \in J \times \mathbb{R}^{N} \times$ $\mathbb{R}^{N} \times \mathbb{R}^{N} \times \mathbb{R}^{N}$, where $q(t) \in C(J, \mathbb{R})$ satisfies $1<q^{-} \leq q^{+}<p^{-} ;$

$\left(2^{0}\right) \lim _{|u|+|v|+|s|+|z| \rightarrow+\infty}\left(e(t, u, v, s, z) /(|u|+|v|+|s|+|z|)^{q(t)-1}\right)=0$, for $t \in J$ uniformly;

$\left(3^{0}\right) \sum_{i=1}^{k}\left|A_{i}(u, v)\right| \leq C_{1}(1+|u|+|v|)^{\theta}$, for all $(u, v) \in \mathbb{R}^{N} \times \mathbb{R}^{N}$, where $0<\theta<\left(p^{-}-1\right) /\left(p^{+}-\right.$ $1)$;

$\left(4^{0}\right) \sum_{i=1}^{k}\left|B_{i}(u, v)\right| \leq C_{2}(1+|u|+|v|)^{\beta-1}$, for all $(u, v) \in \mathbb{R}^{N} \times \mathbb{R}^{N}$, where $1 \leq \beta<q^{+}$;

$\left(5^{0}\right)$ for large enough $R_{0}>0$, the equation

$$
\omega_{\gamma}(l):=\left\{\sum_{\ell=1}^{m-2} \alpha_{\ell}\left[\sum_{t_{i}<\eta_{\ell}} B_{i}(l, 0)+\int_{0}^{\eta_{\ell}} \gamma(t, l, 0, S(l), T(l)) d t\right]\right\}=0,
$$

has no solution on $\partial B\left(R_{0}\right) \cap \mathbb{R}^{N}$, where $B\left(R_{0}\right)=\left\{u \in P C^{1} \mid\|u\|_{1}<R_{0}\right\}$;

$\left(6^{0}\right)$ the Brouwer degree $d_{B}\left[\omega_{\gamma}, b\left(R_{0}\right), 0\right] \neq 0$ for large enough $R_{0}>0$, where $b\left(R_{0}\right)=\{x \in$ $\left.\mathbb{R}^{N}|| x \mid<R_{0}\right\}$.

Then problem (4.18) with (1.2), (1.3), and (1.4) has at least one solution.

Proof. For any $u \in \mathrm{PC}^{1}$ and $\lambda \in[0,1]$, we denote

$$
N_{f_{\lambda}}(u)=\gamma\left(t, u,(w(t))^{1 /(p(t)-1)} u^{\prime}, S(u), T(u)\right)+\lambda e\left(t, u,(w(t))^{1 /(p(t)-1)} u^{\prime}, S(u), T(u)\right) .
$$


At first, we consider the following problem

$$
\begin{gathered}
-\Delta_{p(t)} u+N_{f_{\curlywedge}}(u)(t)=0, \quad t \in(0,1), \quad t \neq t_{i}, \\
\lim _{t \rightarrow t_{i}^{+}} u(t)-\lim _{t \rightarrow t_{i}^{-}} u(t)=A_{i}\left(\lim _{t \rightarrow t_{i}^{-}} u(t), \lim _{t \rightarrow t_{i}^{-}}(w(t))^{1 /(p(t)-1)} u^{\prime}(t)\right), \quad i=1, \ldots, k, \\
\lim _{t \rightarrow t_{i}^{+}} w(t)\left|u^{\prime}\right|^{p(t)-2} u^{\prime}(t)-\lim _{t \rightarrow t_{i}^{-}} w(t)\left|u^{\prime}\right|^{p(t)-2} u^{\prime}(t) \\
=B_{i}\left(\lim _{t \rightarrow t_{i}^{-}} u(t), \lim _{t \rightarrow t_{i}^{-}}(w(t))^{1 /(p(t)-1)} u^{\prime}(t)\right), \quad i=1, \ldots, k, \\
\lim _{t \rightarrow 0^{+}} w(t)\left|u^{\prime}\right|^{p(t)-2} u^{\prime}(t)=\sum_{\ell=1}^{m-2} \alpha_{\ell} \lim _{t \rightarrow \eta_{\ell}^{-}} w(t)\left|u^{\prime}\right|^{p(t)-2} u^{\prime}(t), \quad u(0)=\int_{0}^{1} g(t) u(t) d t .
\end{gathered}
$$

As in the proof of Theorem 4.1, we know that (4.21) has the same solutions of

$$
u=\Psi_{f}^{*}(u, \lambda)=P_{2}(u)+\sum_{t_{i}<t} A_{i}+Q N_{f_{\lambda}}(u)+K_{2}\left(\Theta_{f_{\curlywedge}}(u)\right)
$$

where $\Theta_{f_{\lambda}}$ is defined in (2.39).

We claim that all the solutions of (4.21) are uniformly bounded for $\lambda \in[0,1]$. In fact, if it is false, we can find a sequence of solutions $\left\{\left(u_{n}, \lambda_{n}\right)\right\}$ for (4.21) such that $\left\|u_{n}\right\|_{1} \rightarrow+\infty$ as $n \rightarrow+\infty$, and $\left\|u_{n}\right\|_{1}>1$ for any $n=1,2, \ldots$

Since $\left(u_{n}, \lambda_{n}\right)$ are solutions of $(4.21)$, we have

$$
w(t) \varphi\left(t, u_{n}^{\prime}(t)\right)=\rho_{2}\left(u_{n}\right)+\sum_{t_{i}<t} B_{i}+F\left(N_{f_{\lambda_{n}}}\left(u_{n}\right)\right)(t),
$$

$$
u_{n}(t)=u_{n}(0)+\sum_{t_{i}<t} A_{i}+F\left\{\varphi^{-1}\left[t,(w(t))^{-1}\left(\rho_{2}\left(u_{n}\right)+\sum_{t_{i}<t} B_{i}+F\left(N_{f_{\lambda_{n}}}\left(u_{n}\right)\right)(t)\right)\right]\right\}(t) \text {. Since } u_{n}(0)=
$$
$\int_{0}^{1} g(t) u_{n}(t) d t$, we have

$$
\int_{0}^{1} g(t)\left(F\left\{\varphi^{-1}\left[t,(w(t))^{-1}\left(\rho_{2}\left(u_{n}\right)+\sum_{t_{i}<t} B_{i}+F\left(N_{f_{\lambda_{n}}}\left(u_{n}\right)\right)(t)\right)\right]\right\}(t)+\sum_{t_{i}<t} A_{i}\right) d t=0 .
$$

It follows from Lemma 2.5 that

$$
\left|\rho_{2}\left(u_{n}\right)\right| \leq 3 N C\left(1+\left\|u_{n}\right\|_{1}^{\theta\left(p^{+}-1\right)}+\left\|u_{n}\right\|_{1}^{q^{+}-1}\right) .
$$

From $\left(3^{0}\right),\left(4^{0}\right),(4.23)$ and $(4.25)$, we can see that

$$
\left\|(w(t))^{1 /(p(t)-1)} u_{n}^{\prime}(t)\right\|_{0} \leq o(1)\left\|u_{n}\right\|_{1} .
$$


From (4.26), we have

$$
\lim _{n \rightarrow+\infty} \frac{\left\|u_{n}\right\|_{0}}{\left\|u_{n}\right\|_{1}}=1
$$

Denote $\delta_{n}=\left(\left|u_{n}^{1}\right|_{0} /\left\|u_{n}\right\|_{0},\left|u_{n}^{2}\right|_{0} /\left\|u_{n}\right\|_{0}, \ldots,\left|u_{n}^{N}\right|_{0} /\left\|u_{n}\right\|_{0}\right)$, then $\delta_{n} \in \mathbb{R}^{N}$ and $\left|\delta_{n}\right|=1(n=$ $1,2, \ldots)$. Thus $\left\{\delta_{n}\right\}$ possesses a convergent subsequence (which still denoted by $\delta_{n}$ ), then there exists a vector $\delta_{0}=\left(\delta_{0}^{1}, \delta_{0}^{2}, \ldots, \delta_{0}^{N}\right) \in \mathbb{R}^{N}$ such that $\left|\delta_{0}\right|=1$ and $\lim _{n \rightarrow+\infty} \delta_{n}=\delta_{0}$. Without loss of generality, we assume that $\delta_{0}^{1}>0$. Since $u_{n} \in \mathrm{PC}^{1}$, there exist $\eta_{n}^{i} \in J$ such that

$$
\left|u_{n}^{i}\left(\eta_{n}^{i}\right)\right| \geq\left(1-\frac{1}{n}\right)\left|u_{n}^{i}\right|_{0^{\prime}} \quad i=1,2, \ldots, N ; n=1,2, \ldots
$$

Obviously

$$
\left|u_{n}^{1}(t)-u_{n}^{1}\left(\eta_{n}^{1}\right)\right|=\left|\int_{\eta_{n}^{1}}^{t}\left(u_{n}^{1}\right)^{\prime}(t) d t+\sum_{\eta_{n}^{1}<t_{i}<t} A_{i}^{1}\right| \leq o(1)\left\|u_{n}\right\|_{1} \int_{0}^{1}(w(t))^{-1 /(p(t)-1)} d t+\sum_{i=1}^{k}\left|A_{i}^{1}\right| .
$$
$\left(3^{0}\right)$ that

Note that $\left\|u_{n}\right\|_{1} \rightarrow+\infty$ (as $\left.n \rightarrow+\infty\right)$ and $\delta_{0}^{1}>0$, it follows from (4.27), (4.28), and

$$
\lim _{n \rightarrow+\infty} \frac{\left\{o(1)\left\|u_{n}\right\|_{1} \int_{0}^{1}(w(t))^{-1 /(p(t)-1)} d t+\sum_{i=1}^{k}\left|A_{i}^{1}\right|\right\}}{\left|u_{n}^{1}\left(\eta_{n}^{1}\right)\right|}=0
$$

By (4.27), (4.29), and (4.30) we have $\lim _{n \rightarrow+\infty} u_{n}^{1}(t) / u_{n}^{1}\left(\eta_{n}^{1}\right)=1$ for $t \in J$ uniformly, which implies

$$
\lim _{n \rightarrow+\infty} \frac{u_{n}(t)}{\left\|u_{n}\right\|_{1}}=\delta_{* \prime} \quad \lim _{n \rightarrow+\infty} \frac{(w(t))^{1 /(p(t)-1)} u_{n}^{\prime}(t)}{\left\|u_{n}\right\|_{1}}=0, \quad \text { for } t \in J \text { uniformly, }
$$

where $\delta_{*} \in \mathbb{R}^{N}$, satisfies $\left|\delta_{*}\right|=1,\left|\delta_{*}^{i}\right|=\delta_{0}^{i}$.

From (1.4), we have

$$
\begin{aligned}
0=\sum_{\ell=1}^{m-2} \alpha_{\ell}\left\{\sum_{t_{i}<\eta_{\ell}} B_{i}+\int_{0}^{\eta_{\ell}}[\right. & r\left(t, u_{n},(w(t))^{1 /(p(t)-1)} u_{n}^{\prime}, S\left(u_{n}\right), T\left(u_{n}\right)\right) \\
& \left.\left.+e\left(t, u_{n},(w(t))^{1 /(p(t)-1)} u_{n}^{\prime}, S\left(u_{n}\right), T\left(u_{n}\right)\right)\right] d t\right\} .
\end{aligned}
$$


Note that $\gamma^{1}\left(t, \delta_{*}, 0, S\left(\delta_{*}\right), T\left(\delta_{*}\right)\right) \neq 0$, it follows from $(4.31),\left(4^{0}\right)$ and the continuity of $\gamma^{1}$ that

$$
\sum_{\ell=1}^{m-2} \alpha_{\ell}\left\{\sum_{t_{i}<\eta_{\ell}} B_{i}^{1}+\int_{0}^{\eta_{\ell}}\left\|u_{n}\right\|_{1}^{q(t)-1}\left\{\gamma^{1}\left[t, \delta_{*}, 0, S\left(\delta_{*}\right), T\left(\delta_{*}\right)\right]+o(1)\right\} d t\right\} \neq 0,
$$

which contradicts to (4.32). This implies that there exists a big enough $R_{0}>0$ such that all the solutions of (4.21) belong to $B\left(R_{0}\right)$, then we have

$$
d_{\mathrm{LS}}\left[I-\Psi_{f}^{*}(\cdot, 1), B\left(R_{0}\right), 0\right]=d_{\mathrm{LS}}\left[I-\Psi_{f}^{*}(\cdot, 0), B\left(R_{0}\right), 0\right]
$$

In order to obtaining the existence of solutions (4.18) with (1.2), (1.3), and (1.4), we only need to prove that $d_{\mathrm{LS}}\left[I-\Psi_{f}^{*}(\cdot, 0), B\left(R_{0}\right), 0\right] \neq 0$.

Now we consider the following equation

$$
\begin{gathered}
-\Delta_{p(t)} u+\lambda N_{\gamma}(u)(t)+\frac{(1-\lambda)\left[Q_{N_{\gamma}}(u)\right]}{\sum_{\ell=1}^{m-2} \alpha_{\ell} \eta_{\ell}}=0, \quad t \in(0,1), \quad t \neq t_{i}, \\
\lim _{t \rightarrow t_{i}^{+}} u(t)-\lim _{t \rightarrow t_{i}^{-}} u(t)=\lambda A_{i}\left(\lim _{t \rightarrow t_{i}^{-}} u(t), \lim _{t \rightarrow t_{i}^{-}}(w(t))^{1 /(p(t)-1)} u^{\prime}(t)\right), \quad i=1, \ldots, k, \\
\lim _{t \rightarrow t_{i}^{+}} w(t)\left|u^{\prime}\right|^{p(t)-2} u^{\prime}(t)-\lim _{t \rightarrow t_{i}^{-}} w(t)\left|u^{\prime}\right|^{p(t)-2} u^{\prime}(t) \\
=\lambda B_{i}\left(\lim _{t \rightarrow t_{i}^{-}} u(t), \lim _{t \rightarrow t_{i}^{-}}(w(t))^{1 /(p(t)-1)} u^{\prime}(t)\right), \quad i=1, \ldots, k, \\
\lim _{t \rightarrow 0^{+}} w(t)\left|u^{\prime}\right|^{p(t)-2} u^{\prime}(t)=\sum_{\ell=1}^{m-2} \alpha_{\ell} \lim _{t \rightarrow \eta_{\ell}^{-}} w(t)\left|u^{\prime}\right|^{p(t)-2} u^{\prime}(t), \quad u(0)=\int_{0}^{1} g(t) u(t) d t,
\end{gathered}
$$

where $N_{\gamma}(u)=\gamma\left(t, u,(w(t))^{1 /(p(t)-1)} u^{\prime}, S(u), T(u)\right)$.

Similar to the preceding discussion, for any $\lambda \in(0,1]$, all the solutions of (4.35) are uniformly bounded.

If $u$ is a solution of the following usual equation with (1.4)

$$
\left(w(t)\left|u^{\prime}\right|^{p(t)-2} u^{\prime}\right)^{\prime}=\frac{\left[Q_{N_{r}}(u)\right]}{\sum_{\ell=1}^{m-2} \alpha_{\ell} \eta_{\ell}}, \quad t \in(0,1),
$$

we have

$$
Q_{N_{Y}}(u)=0, \quad w(t)\left|u^{\prime}\right|^{p(t)-2} u^{\prime} \equiv c .
$$


As $u(0)=\int_{0}^{1} g(t) u(t) d t$, we have $w(t)\left|u^{\prime}\right|^{p(t)-2} u^{\prime} \equiv 0$, it means that $u$ is a solution of

$$
\omega_{\gamma}(l)=\left\{\sum_{\ell=1}^{m-2} \alpha_{\ell}\left[\sum_{t_{i}<\eta_{\ell}} B_{i}(l, 0)+\int_{0}^{\eta_{\ell}} \gamma(t, l, 0, S(l), T(l)) d t\right]\right\}=0 .
$$

By hypothesis $\left(5^{0}\right),(4.35)$ has no solutions on $\partial B\left(R_{0}\right) \times[0,1]$, from Theorem 4.1 , we obtain that (4.18) with (1.2), (1.3), and (1.4) has at least one solution. This completes the proof.

Corollary 4.3. If $e: J \times \mathbb{R}^{N} \times \mathbb{R}^{N} \times \mathbb{R}^{N} \times \mathbb{R}^{N} \rightarrow \mathbb{R}^{N}$ is Caratheodory, conditions $\left(2^{0}\right)$, ( $\left.3^{0}\right)$ and $\left(4^{0}\right)$ of Theorem 4.2 are satisfied, condition $\left(3^{0}\right)$ of Corollary 3.6 is also satisfied, $\gamma(t, u, v, S(u), T(u))=$ $\beta(t)\left(|u|^{q(t)-2} u+|v|^{q(t)-2} v+|S(u)|^{q(t)-2} S(u)+|T(u)|^{q(t)-2} T(u)\right)$, where $\beta(t), q(t) \in C(J, \mathbb{R})$ are positive functions satisfying $1<q^{-} \leq q^{+}<p^{-}$; then (4.18) with (1.2), (1.3), and (1.4) has at least one solution.

Proof. Denote

$$
G(l, \lambda)=\left\{\sum_{\ell=1}^{m-2} \alpha_{\ell}\left[\sum_{t_{i}<\eta_{\ell}} \lambda B_{i}(l, 0)+\int_{0}^{\eta_{\ell}} \gamma(t, l, 0, S(l), T(l)) d t\right]\right\} .
$$

From condition $\left(4^{0}\right)$, we have

$$
\left|B_{i}(l, 0)\right| \leq C(1+|l|)^{\beta-1}, \quad 1 \leq \beta<q^{+} .
$$

Note that $k_{*}$ and $h_{*}$ are nonnegative. From the above inequality, we can see that all the solutions of $G(l, \lambda)=0$ are uniformly bounded for $\lambda \in[0,1]$. Thus $d_{B}\left[G(l, \lambda), b\left(R_{0}\right), 0\right]$ is well defined for $\lambda \in[0,1]$ and

$$
\begin{gathered}
d_{B}\left[\omega_{\gamma}, b\left(R_{0}\right), 0\right]=d_{B}\left[G(l, 1), b\left(R_{0}\right), 0\right]=d_{B}\left[G(l, 0), b\left(R_{0}\right), 0\right], \\
G(l, 0)=\left\{\sum_{\ell=1}^{m-2} \alpha_{\ell}\left(\int_{0}^{\eta_{\ell}} \beta(t)\left[|l|^{q(t)-2} l+|S(l)|^{q(t)-2} S(l)+|T(l)|^{q(t)-2} T(l)\right] d t\right)\right\},
\end{gathered}
$$

and it is easy to see that $G(l, 0)=0$ has a unique solution in $\mathbb{R}^{N}$ and

$$
d_{B}\left[\omega_{\gamma}, b\left(R_{0}\right), 0\right]=d_{B}\left[I, b\left(R_{0}\right), 0\right] \neq 0 .
$$

According to Theorem 4.2, we get that (4.18) with (1.2), (1.3), and (1.4) has at least a solution. This completes the proof.

Let us consider

$$
-\Delta_{p(t)} u+f\left(t, u,(w(t))^{1 /(p(t)-1)} u^{\prime}, S(u), T(u), \delta\right)=0, \quad t \in(0,1), t \neq t_{i}
$$


where $\delta$ is a parameter, and

$$
\begin{aligned}
& f\left(t, u,(w(t))^{1 /(p(t)-1)} u^{\prime}, S(u), T(u), \delta\right) \\
& \quad=s\left(t, u,(w(t))^{1 /(p(t)-1)} u^{\prime}, S(u), T(u)\right)+\delta h\left(t, u,(w(t))^{1 /(p(t)-1)} u^{\prime}, S(u), T(u)\right),
\end{aligned}
$$

where $h, \varsigma: J \times \mathbb{R}^{N} \times \mathbb{R}^{N} \times \mathbb{R}^{N} \times \mathbb{R}^{N} \rightarrow \mathbb{R}^{N}$ are Caratheodory.

From Theorem 4.2, similar to the proof of Theorem 3.3, we have the following.

Theorem 4.4. If conditions of $\left(1^{0}\right)$ and $\left(3^{0}\right)-\left(6^{0}\right)$ of Theorem 4.2 are satisfied, then problem (4.43) with (1.2), (1.3), and (1.4) has at least one solution when the parameter $\delta$ is small enough.

Theorem 4.5. If conditions of $\left(1^{0}\right)-\left(3^{0}\right)$ and $\left(5^{0}\right)-\left(6^{0}\right)$ of Theorem 4.2 are satisfied, and $D$ satisfy

$$
\sum_{i=1}^{k}\left|D_{i}(u, v)\right| \leq C(1+|u|+|v|)^{\alpha_{i}^{+}}, \quad \forall(u, v) \in \mathbb{R}^{N} \times \mathbb{R}^{N}
$$

where

$$
\alpha_{i} \leq \frac{q^{+}-1}{p\left(r_{i}\right)-1}, \quad p\left(r_{i}\right)-1 \leq q^{+}-\alpha_{i}, \quad i=1, \ldots, k
$$

then problem (4.18) with (1.2), (1.3), and (1.8) has at least one solution.

Proof. Similar to the proof of Theorem 3.2, the condition $\left(4^{0}\right)$ of Theorem 4.2 is satisfied. Thus problem (4.18) with (1.2), (1.3) and (1.8) has at least a solution.

Similar to the proof of Theorem 3.2 and Corollary 4.3, we have the following.

Corollary 4.6. If $e: J \times \mathbb{R}^{N} \times \mathbb{R}^{N} \times \mathbb{R}^{N} \times \mathbb{R}^{N} \rightarrow \mathbb{R}^{N}$ is Caratheodory, (4.45), (4.46) and conditions $\left(2^{0}\right)$ and $\left(3^{0}\right)$ of Theorem 4.2 are satisfied, condition $\left(3^{0}\right)$ of Corollary 3.6 is also satisfied, $\gamma(t, u, v, S(u), T(u))=\beta(t)\left(|u|^{q(t)-2} u+|v|^{q(t)-2} v+|S(u)|^{q(t)-2} S(u)+|T(u)|^{q(t)-2} T(u)\right)$, where $\beta(t), q(t) \in C(J, \mathbb{R})$ are positive functions satisfying $1<q^{-} \leq q^{+}<p^{-}$; then (4.43) with (1.2), (1.3), and (1.8) has at least one solution when the parameter $\delta$ is small enough.

\section{Acknowledgments}

This paper is partly supported by the National Science Foundation of China (10701066, 10926075, and 10971087), China Postdoctoral Science Foundation funded project (20090460969), and the Natural Science Foundation of Henan Education Committee (2008-755-65).

\section{References}

[1] E. Acerbi and G. Mingione, "Regularity results for stationary electro-rheological fluids," Archive for Rational Mechanics and Analysis, vol. 164, no. 3, pp. 213-259, 2002.

[2] Y. Chen, S. Levine, and M. Rao, "Variable exponent, linear growth functionals in image restoration," SIAM Journal on Applied Mathematics, vol. 66, no. 4, pp. 1383-1406, 2006. 
[3] M. Růžička, Electrorheological Fluids: Modeling and Mathematical Theory, vol. 1748 of Lecture Notes in Mathematics, Springer, Berlin, Germany, 2000.

[4] V. V. Zhikov, "Averaging of functionals of the calculus of variations and elasticity theory," Mathematics of the USSR-Izvestiya, vol. 29, pp. 33-36, 1986.

[5] E. Acerbi and G. Mingione, "Regularity results for a class of functionals with non-standard growth," Archive for Rational Mechanics and Analysis, vol. 156, no. 2, pp. 121-140, 2001.

[6] A. Coscia and G. Mingione, "Hölder continuity of the gradient of $p(x)$-harmonic mappings," Comptes Rendus de l'Académie des Sciences. Série I. Mathématique, vol. 328, no. 4, pp. 363-368, 1999.

[7] S.-G. Deng, "A local mountain pass theorem and applications to a double perturbed $p(x)$-Laplacian equations," Applied Mathematics and Computation, vol. 211, no. 1, pp. 234-241, 2009.

[8] X. Fan, "Global $C^{1, \alpha}$ regularity for variable exponent elliptic equations in divergence form," Journal of Differential Equations, vol. 235, no. 2, pp. 397-417, 2007.

[9] X. Fan, "Boundary trace embedding theorems for variable exponent Sobolev spaces," Journal of Mathematical Analysis and Applications, vol. 339, no. 2, pp. 1395-1412, 2008.

[10] X. Fan, Q. Zhang, and D. Zhao, "Eigenvalues of $p(x)$-Laplacian Dirichlet problem," Journal of Mathematical Analysis and Applications, vol. 302, no. 2, pp. 306-317, 2005.

[11] P. Harjulehto, P. Hästö, and V. Latvala, "Harnack's inequality for $p(\cdot)$-harmonic functions with unbounded exponent $p$, " Journal of Mathematical Analysis and Applications, vol. 352, no. 1, pp. 345-359, 2009.

[12] M. Mihăilescu and V. Rădulescu, "Continuous spectrum for a class of nonhomogeneous differential operators," Manuscripta Mathematica, vol. 125, no. 2, pp. 157-167, 2008.

[13] M. Mihăilescu, P. Pucci, and V. Rădulescu, "Nonhomogeneous boundary value problems in anisotropic Sobolev spaces," Comptes Rendus de l'Académie des Sciences-Series I, vol. 345, no. 10, pp. 561-566, 2007.

[14] M. Mihăilescu, V. Rădulescu, and D. Repovš, “On a non-homogeneous eigenvalue problem involving a potential: an Orlicz-Sobolev space setting," Journal de Mathématiques Pures et Appliquées, vol. 93, no. 2, pp. 132-148, 2010.

[15] J. Musielak, Orlicz Spaces and Modular Spaces, vol. 1034 of Lecture Notes in Mathematics, Springer, Berlin, Germany, 1983.

[16] S. G. Samko, "DensityC $C_{0}^{\infty}\left(\mathbb{R}^{N}\right)$ in the generalized Sobolev spaces $W^{m, p(x)}\left(\mathbb{R}^{N}\right)$," Doklady Rossiuskaya Akademiya Nauk, vol. 369, no. 4, pp. 451-454, 1999.

[17] Q. Zhang, "Existence of solutions for $p(x)$-Laplacian equations with singular coefficients in $\mathbb{R}^{N}$," Journal of Mathematical Analysis and Applications, vol. 348, no. 1, pp. 38-50, 2008.

[18] I.-S. Kim and Yun-Ho Kim, "Global bifurcation of the $p$-Laplacian in $\mathbb{R}^{N}$," Nonlinear Analysis: Theory, Methods E Applications, vol. 70, no. 7, pp. 2685-2690, 2009.

[19] B. Ahmad and J. J. Nieto, "The monotone iterative technique for three-point second-order integrodifferential boundary value problems with $p$-Laplacian," Boundary Value Problems, Article ID 57481, 9 pages, 2007.

[20] J. Jiao, L. Chen, and L. Li, "Asymptotic behavior of solutions of second-order nonlinear impulsive differential equations," Journal of Mathematical Analysis and Applications, vol. 337, no. 1, pp. 458-463, 2008.

[21] J. Li, J. J. Nieto, and J. Shen, "Impulsive periodic boundary value problems of first-order differential equations," Journal of Mathematical Analysis and Applications, vol. 325, no. 1, pp. 226-236, 2007.

[22] L. Liu, L. Hu, and Y. Wu, "Positive solutions of two-point boundary value problems for systems of nonlinear second-order singular and impulsive differential equations," Nonlinear Analysis: Theory, Methods \& Applications, vol. 69, no. 11, pp. 3774-3789, 2008.

[23] J. J. Nieto and D. O'Regan, "Variational approach to impulsive differential equations," Nonlinear Analysis: Real World Applications, vol. 10, no. 2, pp. 680-690, 2009.

[24] J. J. Nieto, "Impulsive resonance periodic problems of first order," Applied Mathematics Letters, vol. 15, no. 4, pp. 489-493, 2002.

[25] L. Di Piazza and B. Satco, "A new result on impulsive differential equations involving non-absolutely convergent integrals," Journal of Mathematical Analysis and Applications, vol. 352, no. 2, pp. 954-963, 2009.

[26] J. Shen and W. Wang, "Impulsive boundary value problems with nonlinear boundary conditions," Nonlinear Analysis: Theory, Methods E Applications, vol. 69, no. 11, pp. 4055-4062, 2008. 
[27] M. Yao, A. Zhao, and J. Yan, "Periodic boundary value problems of second-order impulsive differential equations," Nonlinear Analysis: Theory, Methods E Applications, vol. 70, no. 1, pp. 262-273, 2009.

[28] M. Feng, B. Du, and W. Ge, "Impulsive boundary value problems with integral boundary conditions and one-dimensional p-Laplacian," Nonlinear Analysis: Theory, Methods \& Applications, vol. 70, no. 9, pp. 3119-3126, 2009.

[29] A. Kristály, V. Rădulescu, and C. Varga, Variational Principles in Mathematical Physics, Geometry, and Economics: Qualitative Analysis of Nonlinear Equations and Unilateral Problems, Encyclopedia of Mathematics and its Applications, No. 136, Cambridge University Press, Cambridge, UK, 2010.

[30] A. Cabada and J. Tomeček, "Extremal solutions for nonlinear functional $\phi$-Laplacian impulsive equations," Nonlinear Analysis: Theory, Methods E Applications, vol. 67, no. 3, pp. 827-841, 2007.

[31] Q. Zhang, Z. Qiu, and X. Liu, "Existence of solutions and nonnegative solutions for weighted $p(r)$-Laplacian impulsive system multi-point boundary value problems," Nonlinear Analysis: Theory, Methods \& Applications, vol. 71, no. 9, pp. 3814-3825, 2009.

[32] Z. Yang, "Existence of nontrivial solutions for a nonlinear Sturm-Liouville problem with integral boundary conditions," Nonlinear Analysis: Theory, Methods E Applications, vol. 68, no. 1, pp. 216-225, 2008.

[33] Y. Li and F. Li, "Sign-changing solutions to second-order integral boundary value problems," Nonlinear Analysis: Theory, Methods \& Applications, vol. 69, no. 4, pp. 1179-1187, 2008.

[34] R. Ma and Y. An, "Global structure of positive solutions for nonlocal boundary value problems involving integral conditions," Nonlinear Analysis: Theory, Methods \& Applications, vol. 71, no. 10, pp. 4364-4376, 2009.

[35] X. Zhang, X. Yang, and W. Ge, "Positive solutions of $n$ th-order impulsive boundary value problems with integral boundary conditions in Banach spaces," Nonlinear Analysis: Theory, Methods $\mathcal{E}$ Applications, vol. 71, no. 12, pp. 5930-5945, 2009. 